\title{
The first search for variable stars in the open cluster NGC 6253 and its surrounding field ${ }^{\star}, \star \star$
}

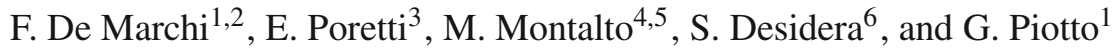

\author{
1 Dipartimento di Astronomia, Università di Padova, Vicolo dell'Osservatorio 2, 35122 Padova, Italy \\ e-mail: fdemarchi@science.unitn.it \\ 2 Dipartimento di Fisica, Università di Trento, Via Sommarive 14, 38123 Povo (TN), Italy \\ 3 INAF - Osservatorio Astronomico di Brera, Via E. Bianchi 46, 23807 Merate (LC), Italy \\ 4 Universitaets-Sternwarte der Ludwig-Maximilians-Universitaet, Scheinerstr. 1, 81679 Muenchen, Germany \\ 5 Max-Planck-Institute for Extraterrestrial Physics, Giessenbachstr., 85748 Garching bei Muenchen, Germany \\ ${ }^{6}$ INAF - Osservatorio Astronomico di Padova, Vicolo dell'Osservatorio 5, 35122 Padova, Italy
}

Received 22 May 2009 / Accepted 30 September 2009

\section{ABSTRACT}

\begin{abstract}
Aims. This work presents the first high-precision variability survey in the field of the intermediate-age, metal-rich open cluster NGC 6253. Clusters of this type are benchmarks for stellar evolution models.

Methods. Continuous photometric monitoring of the cluster and its surrounding field was performed over a time span of ten nights using the Wide Field Imager mounted at the ESO-MPI $2.2 \mathrm{~m}$ telescope. High-quality timeseries, each composed of about 800 datapoints, were obtained for 250000 stars using ISIS and DAOPHOT packages. Candidate members were selected by using the colour-magnitude diagrams and period-luminosity-colour relations. Membership probabilities based on the proper motions were also used. The membership of all the variables discovered within a radius of $8^{\prime}$ from the centre is discussed by comparing the incidence of the classes in the cluster direction and in the surrounding field.

Results. We discovered 595 variables and we also characterized most of them providing their variability classes, periods, and amplitudes. The sample is complete for short periods: we classified 20 pulsating variables, 225 contact systems, 99 eclipsing systems (22 $\beta$ Lyr type, $59 \beta$ Per type, 18 RS CVn type), and 77 rotational variables. The time-baseline hampered the precise characterization of 173 variables with periods longer than 4-5 days. Moreover, we found a cataclysmic system undergoing an outburst of about 2.5 mag. We propose a list of 35 variable stars as probable members of NGC 6253 .
\end{abstract}

Key words. starspots - stars: statistics - stars: variables: general - binaries: eclipsing - novae, cataclysmic variables open clusters and associations: individual: NGC 6253

\section{Introduction}

NGC 6253 and NGC 6791 are the only open clusters whose metallicities above $[\mathrm{Fe} / \mathrm{H}]=+0.3$ were confirmed by spectroscopic analyses (Carretta et al. 2000, 2007; Sestito et al. 2007). Therefore, these clusters are of special interest in several fields, e.g., as benchmarks for stellar evolution and stellar population models and as targets for the search for extrasolar planets. We observed both clusters in the framework of our project looking for transiting planets in super-metal-rich open clusters. The results obtained on NGC 6791 were presented by Montalto et al. (2007).

We also performed a 10-night observing campaign on NGC 6253 with the same purposes as for NGC 6791. In the first paper based on our new investigation, Montalto et al. (2009) obtained broad band photometry and astrometry for 187963 stars within 30 arcmin from the cluster. Images from ESO archive

^ Based on observation made at the European Southern Observatory, La Silla, Chile, Proposal 073.C-0227.

$\star \star$ Timeseries and light curves are available in electronic form at the CDS via anonymous ftp to

cdsarc.u-strasbg.fr $(130.79 .128 .5)$ or via

http://cdsweb.u-strasbg.fr/cgi-bin/qcat?J/A+A/509/A17
(Momany et al. 2001) were also used to derive relative proper motions and then distinguish between field stars and cluster members. The availability of the astrometric cluster memberships and the photometric quality of the new data allowed new, independent determinations of the cluster's main parameters. Indeed, the determinations of the NGC 6253 parameters are affected by larger uncertainties because of the cluster's projection toward a very rich stellar field fairly close to the galactic centre ( $l=335.46 \mathrm{deg}, b=-6.25 \mathrm{deg})$. Systematic differences in the photometric calibrations of different datasets have been found (Bragaglia et al. 1997; Piatti et al. 1998; Sagar et al. 2001; Twarog et al. 2003; Anthony-Twarog et al. 2007). In this paper we adopt the values of the distance modulus and of the reddenings obtained by Montalto et al. (2009) using the technique of the isochrone fitting, i.e., $(m-M)_{V}=11.68 \pm 0.10 \mathrm{mag}, E(B-V)=$ $0.15 \pm 0.02 \mathrm{mag}$ and $E(V-I)=0.25 \pm 0.02 \mathrm{mag}$. These values are also consistent with a weighted mean of all the determinations. The cluster age is about 3.5 Gyr (Montalto et al. 2009).

Our project gives the possibility of studying stellar variability in super-metal-rich stars using high-quality data (De Marchi et al. 2007). Since no variability survey on NGC 6253 has previously been performed, we characterize the variable stars in NGC 6253 and in its surrounding field for the first time. To do 
Table 1. The observation $\log$ for each night and limits of the field of view.

\begin{tabular}{ccc|ccc}
\hline \hline $\begin{array}{c}\text { Date } \\
\text { [Year 2004] }\end{array}$ & $\begin{array}{c}t_{\text {start }} \\
\text { [HJD-2 453 100] }\end{array}$ & $\begin{array}{c}t_{\text {end }} \\
\text { [Year 2004] }\end{array}$ & $\begin{array}{c}t_{\text {start }} \\
\text { [HJD-2 253 100] }\end{array}$ \\
\hline June, 13-14 & 70.57 & 70.91 & June, 18-19 & 75.48 & 75.90 \\
June, 14-15 & 71.49 & 71.91 & June, 19-20 & 76.46 & 76.67 \\
June, 15-16 & 72.46 & 72.90 & June, 20-21 & 77.84 & 77.87 \\
June, 16-17 & 73.69 & 73.87 & June, 21-22 & 78.44 & 78.76 \\
June, 17-18 & 74.49 & 74.89 & June, 22-23 & 79.46 & 79.90 \\
\hline$\alpha_{\min }$ & $16^{\mathrm{h}} 56^{\mathrm{m}} 41.6$ & $\alpha_{\max }$ & $17^{\mathrm{h}} 00^{\mathrm{m}} 24.7$ \\
$\delta_{\min }$ & $-53^{\circ} 05^{\prime} 43.8^{\prime \prime}$ & $\delta_{\max }$ & $-52^{\circ} 33^{\prime} 00.8^{\prime \prime}$ \\
\hline
\end{tabular}

that, we started from the new findings and calibrations obtained by Montalto et al. (2009) so we refer the reader to that paper for a more detailed explanation of the methodologies applied to determine the properties and the fundamental parameters of the cluster.

\section{Observations and data reduction}

NGC 6253 was observed for 10 consecutive nights (from June 13, 2004 to June 22, 2004) using the Wide-Field Imager (WFI) mounted at the ESO-MPI $2.2 \mathrm{~m}$ telescope, La Silla, Chile. The WFI instrument includes a mosaic of eight $2 \mathrm{k} \times 4 \mathrm{k}$ CCDs. The pixel scale is 0.238 arcsec/pixel. In total, $\sim 45.3 \mathrm{~h}$ of observation were collected, mainly in the $R$ filter. A few deep images in the $B, V$, and $I$ filters were also acquired to construct colour-magnitude diagrams (CMDs), along with a standard field to allow the calibration of the data. In total $918 \mathrm{im}-$ ages of the cluster were obtained, with a mean exposure time of $178 \mathrm{~s}$. Table 1 reports the journal of observations and Fig. 1 shows a WFI image of NGC 6253. Since the the size of each chip is $8^{\prime}$ in right ascension and $16^{\prime}$ in declination, we centered the cluster on one chip to minimize the loss of stars between chips. Observations and data reduction to derive the calibrated photometry and the CMDs of the cluster are described in more detail in Montalto et al. (2009). The procedure to derive the light curves uses both ISIS 2.2 (Alard \& Lupton 1998; Alard 2000) and DAOPHOT II (Stetson 1998) packages, as described in Montalto et al. (2007).

The length of the observing nights (more than $0.32 \mathrm{~d}$ in 7 cases and more than $0.40 \mathrm{~d}$ in 5 cases, see Table 1) reduced the height of the aliases situated at $\pm 1 \mathrm{~d}^{-1}$ from the central peak down to below $60 \%$ of the power (Fig. 2, upper panel). Moreover, the light curves are very dense and their shape clearly defined on each night (Fig. 2, middle panel). Both these facts made the period detection quite straightforward, not only in the case of high-amplitude variables, but most of time also for smallamplitude, short-period variable stars.

As can be noted in Fig. 1, our survey covers a much larger field of view than the previous ones $\left(6.3 \times 6.3 \mathrm{arcmin}^{2}\right.$ by Bragaglia et al. $1997,3.8 \times 3.8 \operatorname{arcmin}^{2}$ by Piatti et al. 1998). We could also identify new variable stars in a wide part of the surrounding field. The ISIS 2.2. and DAOPHOT II packages returned a photometric precision well below $0.01 \mathrm{mag}$ in the magnitude range $14 \leq R \leq 19$. A plot of the standard errors of the mean magnitudes in different filters is shown in Fig. 1 in Montalto et al. (2009). Stars brighter than the turn-off magnitude $(V=14.5)$ are saturated in our photometry and cannot be studied. In particular, this constraint hampers the study of the variability of the blue stragglers, as performed by De Marchi (2008) in the more favourable case of NGC 6791.

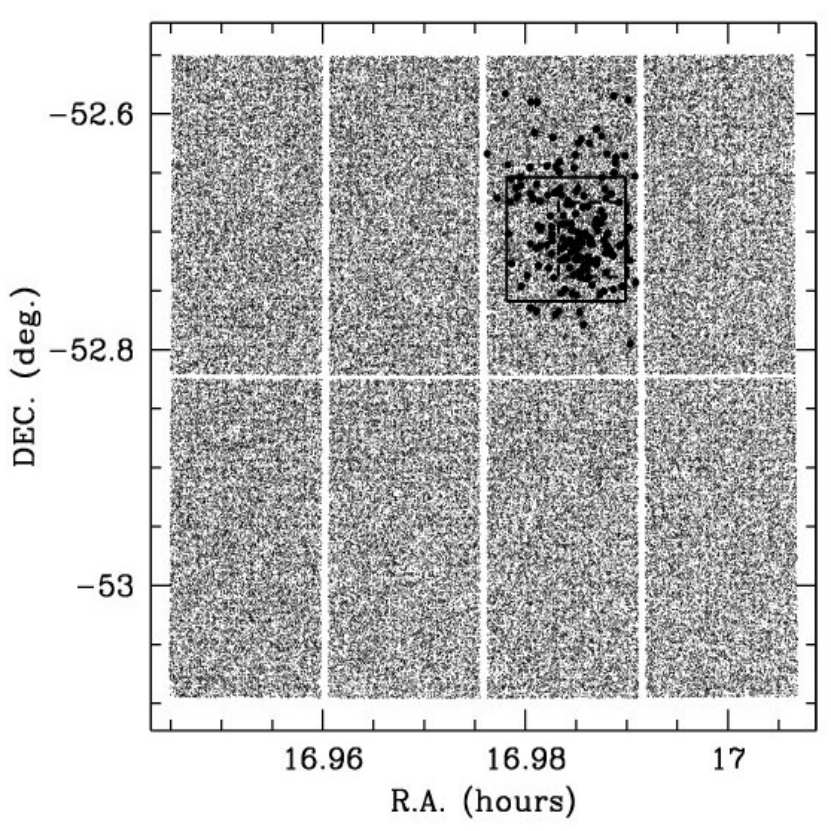

Fig. 1. Image of the WFI field $\left(32 \times 32 \mathrm{arcmin}^{2}\right)$. Solid lines represent the edges of the $6.3 \times 6.3 \operatorname{arcmin}^{2}$ box surveyed by Bragaglia et al. (1997). Large points are stars with membership probabilities (available only for stars located in chip 2) greater than $90 \%$. Chips are numbered from 1 (top right) to 8 (bottom right).
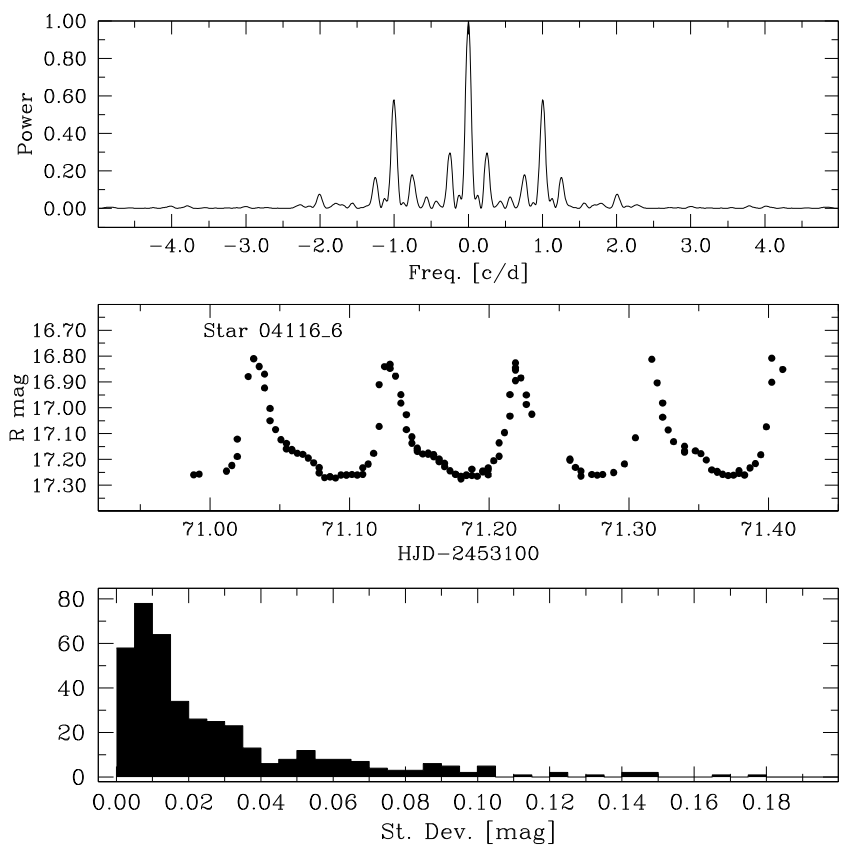

Fig. 2. Upper panel: spectral window of the timeseries of the variable stars in NGC 6253. Middle panel: example of an unfolded light curve: the high-amplitude $\delta$ Sct star 044116_6. Bottom panel: histograms of the standard deviations of the least-squares fit on the light curves of the periodic variables.

\section{Cluster membership}

NGC 6253 is a relatively small cluster, but Bragaglia et al. (1997) noticed the necessity of moving $8^{\prime}$ from the cluster centre to find a legitimate external field. We followed this prescription and we adopted the centre coordinates given by Bragaglia et al. (1997). 
The measured stars are indicated by small points, the $\sim 150$ stars with membership probability (hereafter MP) greater than $90 \%$ are highlighted with larger black points. MPs were calculated in Montalto et al. (2009) following the approach proposed by Vasilevskis et al. (1958):

$M P=\Phi_{\mathrm{c}} /\left(\Phi_{\mathrm{c}}+\Phi_{\mathrm{f}}\right)$

where $\Phi_{\mathrm{c}}$ and $\Phi_{\mathrm{f}}$ are the distribution of cluster and field stars in the diagram of the proper motions, respectively. These distributions are typically represented as Gaussian functions. The distribution of the cluster stars has a narrow peak centered at $\mu_{\alpha}=\mu_{\delta}=0$, while the distribution of field stars is much broader. For the given candidate member, the calculation of the MP was performed by selecting a surrounding sample of a 2.5 mag range centered on the candidate's position. In such a way the local sample stars compensate for the effect of a magnitude dependence of the cluster-to-field star ratio. When constructing a $V$ - MP diagram, the stars belonging to the cluster occupy a well-defined region (see Fig. 4 in Montalto et al. 2009). We require the probable member clusters to have MP $>90 \%$ at $V=12.5$ and MP $>50 \%$ at $V=18.0$.

Since the determination of the MPs is a differential process and the cluster is almost completely included in chip 2, the MPs are reliable only for stars belonging to this chip and brighter than $V=18$. Looking at the distribution of the stars with a high MP we can infer that some members of the cluster might also be present in chips 1 and 3 .

\section{The variable stars}

\subsection{Detection}

The ISIS 2.2 and DAOPHOT II packages allowed us to extract the first list of suspected variable stars from the full database of 250000 timeseries. This list was validated and shortened by calculating the parameters related to the reduction of the initial variance obtained by introducing trial periodic terms. These parameters are the reduction factor (Vaníček 1971) and the coefficient of spectral correlation (Ferraz-Mello 1981). As in the case of NGC 6971 (De Marchi et al. 2007), we could separate shortand long-period variable stars by introducing a parameter that is more sensitive to the night-to-night variations. Tests on the significance of the detected periodicities (e.g., signal-to-noise ratio above 4.0 in amplitude) allowed us to get a more defined sample of real variable stars. A few objects whose variability appears to stem from photometric artefacts (e.g. eclipse-like features occurring exactly at the same time on the second night) were removed from the list. These spurious photometric effects are usually corrected when applying to the light-curve algorithms such as the one developed by Tamuz et al. (2005). However, we noticed that the application of this algorithm degrades the precision of the variable star photometry. Therefore, being interested in much greater light variations than the tiny photometric effect of a planetary transit, we decided to analyse the light curves before applying the algorithm.

We identified 595 variable stars at the end of our process, whose timeseries are composed of about 800 datapoints. To identify them we used the five-digit number assigned by our customized package package, followed by the number of the chip that the star belongs to. The timeseries are available at the "Centre de données astronomiques de Strasbourg" (CDS).
Table 2. Inventory of the variables found in NGC 6253 and its surrounding area.

\begin{tabular}{lcccc}
\hline \hline Type & all chips & $\begin{array}{c}\text { Number of variables } \\
r<8^{\prime}\end{array}$ & $\begin{array}{c}\text { candidate } \\
\text { members }\end{array}$ & $\begin{array}{c}\text { probable } \\
\text { members }\end{array}$ \\
\hline RR Lyrae & 4 & 1 & 0 & 0 \\
$\delta$ Scuti & 11 & 0 & 0 & 0 \\
$\beta$ Cep & 1 & 0 & 0 & 0 \\
HADS & 4 & 0 & 0 & 0 \\
EW-type & 225 & 50 & 16 & 8 \\
EB-type & 22 & 6 & 2 & 0 \\
EA-type & 59 & 13 & 5 & 1 \\
RS CVn & 18 & 4 & 2 & 1 \\
U Geminorum & 1 & 1 & 1 & 1 \\
Rotationals & 77 & 27 & 16 & 15 \\
Long period & 173 & 41 & 16 & 9 \\
\hline
\end{tabular}

\subsection{Classification}

The timeseries of the 595 variable stars were analysed in frequency by using the least-squares iterative sine-wave search (Vaníček 1971) and the Phase Dispersion Minimization (Stellingwerf 1978) methods. The periods were refined by means of a least-squares procedure (MTRAP, Carpino et al. 1987); their error bars are in the range $1-6 \times 10^{-5} \mathrm{~d}$. The bottom panel of Fig. 2 shows the distribution of the standard deviations of the least-squares fits, indicating a median precision of $0.015 \mathrm{mag}$.

We could show amplitudes of light variability down to the $0.01 \mathrm{mag}$ level. At this level, rotational variables could be separated from pulsating variables on the basis of the period values and of the Fourier parameters alone (Poretti 2001). On the other hand, it is very difficult to disentangle rotational from eclipsing variables. To distinguish rotational variables from contact binaries, we referred to the degree of asymmetry of the double-wave light curves and to the occurrence of the minima at phases 0.00 and 0.50 . Of course, we cannot rule out that a small fraction of the variables classified as rotational variables might be actually contact systems showing grazing eclipses or viceversa.

We considered two classes of rotational variables, RO1 and RO2 stars. RO1 stars show a light curve characterized by a single wave, which is often asymmetrical. RO2 stars show a more complicated curve composed of two waves having unequal amplitude and duration. This light curve is comes from two (groups of) spots located at different latitudes that remain visible to the observer during different fractions of the rotational period. In some cases these spotted stars are observed in eclipsing systems, the so-called RS CVn variables. Other cases of eclipsing systems are contact (W UMa variables, EW), semi-detached ( $\beta$ Lyr variables, EB), and detached systems (Algol variables, EA) binaries. In some cases it was very difficult to distinguish between EW system showing grazing eclipses and rotational variables. We also identified three different classes of pulsating variables, i.e., RR Lyr, $\delta$ Sct and high-amplitude $\delta$ Sct (HADS) stars. In both cases, eclipsing binaries and pulsating variables, the very good spectral window (Fig. 2) made the period detection quite straightforward. On the other hand, defining the periods longer than 4-5 d was not easy. In particular it was impossible for periods longer than $10 \mathrm{~d}$ and we simply classify these stars as long period (LON) variables. These stars are mostly rotational variables. The summary classification of the entire sample is reported in Table 2. Tables A.1-A.8 list the members of each class giving the identifier in the Montalto et al. (2007) catalogue, the coordinates, the photometry, the epoch of maximum or 
minimum brightness (HJD-2 453 100), the period, the amplitude, the distance, and the MP values. Uncertain MP values (stars with $V>18$, often close to the chip borders) are marked with an asterisk. The catalogue of the light curves of the periodic variables is available at the CDS.

We paid particular attention to the variables located within $8^{\prime}$ from the cluster centre; in any cases no variable with MP larger than $50 \%$ was found at a greater distance. If the MP is not available (stars near the edges of chip 2 or stars fainter than $V=18$ ), the membership is estimated from their location on the $B-V$ vs. $V$ and $V-I$ vs. $V$ CMDs. Moreover, for pulsating variables and contact binaries with unknown membership, our conclusions are based on the applications of the usual period-luminosity $(P-L)$ and period-luminosity-colour $(P-L-C)$ relations.

\subsection{Pulsating variables}

The only pulsating variable located at less than $8^{\prime}$ from the centre is the RRab star 10540_2. Its MP is quite high, but it is clearly too faint ( $V=17.39$ ) to belong to the cluster. Amongst the other four RR Lyr stars, 15578_7 is a new galactic Blazhko variable.

Twelve variables show an amplitude smaller than 0.06 mag; since they have a very short period (less than $0.10 \mathrm{~d}$ ), we can rule out their being rotational variables. All the $B-V$ values except one range from 0.42 to 0.79 mag, mostly between 0.50 and 0.63 . This interval, taking the reddening $E(B-V)=0.15$ mag into account, suggests their classification as $\delta$ Sct stars. The remaining low-amplitude variable shows $(B-V)=0.084$ : it probably belongs to the $\beta$ Cep class.

Four variables show a larger amplitude (more than $0.09 \mathrm{mag}$ ) and the asymmetric shape of the light curve typical for highamplitude $\delta$ Sct stars. By using the new period-luminosity relation derived by Poretti et al. (2008), no doubt is left on the fact that all these variables do not belong to NCG 6253. Finally, none of the pulsating variables is a member of NGC 6253, since they are all located well beyond the cluster.

\subsection{Contact binaries}

We have 50 contact binaries located within the $8^{\prime}$ radius and the $(B-V)$ and $(V-I)$ colours are both available for 44 binaries, while only the $(V-I)$ colours are available for 6 of them. For these stars it is possible to apply the $P-L-C$ relations given by Rucinski (2003) and compare the resulting distance moduli with that of the cluster, obtained by isochrone fitting (Montalto et al. 2009). The errors on the distance moduli are calculated by considering the uncertainties on the mean $B-V$ and $V-I$ colours. It must also be taken into account that, since our light curves are in the $R$ band, we cannot know the exact value $V_{\max }$ of the magnitude at maximum brightness required by the Rucinski (2003) calibrations. We estimated $V_{\max }$ as $\left(V_{\text {mean }}-R_{\text {mean }}\right)+R_{\max }$; i.e., we assumed that the colour of these binary systems does not change during the orbital period since the components have a very similar temperature.

An estimate of the membership of the objects can be obtained using the CMDs (Fig. 3, top row), the MPs based on the proper motions, and the two $P-L-C$ relations (Fig. 3, bottom row). The fiducial lines shown in the CMDs are obtained by selecting $10-15$ points at different magnitudes along the Main Sequences, both from the observations described here and from Bragaglia et al. (1997). We could select a list of 13 candidate members for which one of the above criteria is satisfied (Table A.9).
We note that 23188_2 and 10853_2 satisfy all the membership criteria and then are very likely cluster members. The position in the CMDs and the $P-L-C$ relations also suggest that $01015 \_2$ is a cluster member, but this hint is not supported by the MP, which is very small. The case of 09268_2 is the opposite: it has also a fairly large MP, but the other indicators suggest that is is more probably located between the Sun and the cluster. Unfortunately, none of the remaining cases gives us enough confidence on a cluster membership.

We can tackle the problem of cluster membership in an indirect way. In the surrounding field we found $175 \mathrm{EW}$ binaries, with an incidence of $0.21 \mathrm{EW} \operatorname{arcmin}^{-2}$. Therefore, we should have 42 field EW-stars superposed on the cluster. Since we found 50 stars (Table 2), the excess is only marginally significant. We have only two well-established memberships; therefore, we can reasonably estimate that very few contact binaries (up to six) among the remaining 11 candidates listed in Table A.9 actually belong to NGC 6253. This clue is confirmed by the candidates do not match the photometric criteria very well (Table A.9). In NGC 6791 we found three well-established and five likely EW-members (De Marchi et al. 2007), i.e., similar countings. The surveys of the two clusters are complete both at the magnitude and at the periods of the EW binaries. The two clusters have a different stellar content, since NGC 6253 has about 500-1000 members (Montalto et al. 2009), and NGC 6791 about $4900 \pm 1000$ (De Marchi 2008). The similarity between EW countings in the two clusters supports the hypothesis of an anticorrelation between the frequency of binaries and the richness of the host cluster (Kaluzny \& Rucinski 1995).

Among the non-member contact binaries, we note that 00441_4 has a period of $0.21002 \mathrm{~d}$, shorter than the shortest contact binary found in the ASAS database $(P=0.217811 \mathrm{~d}$, ASAS 083128+1953.1, Rucinski 2007) and very similar to the binary with the shortest period known $(P=0.2009 \mathrm{~d}$, V344 in the Lupus field, Weldrake \& Bayliss 2008).

\subsection{Semi-detached and detached systems}

The sample of the semi-detached and detached systems within $8^{\prime}$ is composed of five EA, two EB, and two RS CVn stars (lower part of Table A.9). Their periods are shorter than $2.3 \mathrm{~d}$. The star 26902_2 has a high MP, and it is the only case for which we can be very confident about its membership, also confirmed by the positions in the CMDs (Fig. 4, left panels). On the basis of the same criteria, 10340_2 is another probable member. On the other hand, the MP value rules out the membership of 00145_2. No firm conclusion on the membership can be drawn on the other cases.

\subsection{Rotational and long-period variables}

A great number of the new variables discovered in our survey shows the single (RO1) or double (RO2) wave light curves typical of rotational effect. The 10-d time baseline allowed us to detect all the variables with rotational periods shorter than 4-5 days. Other variables show an evident night-to-night variability, but we cannot infer any reliable value for the period. These variables are probably long-period ones (LON).

By adopting the same criteria as used in other cases, we selected the RO1 (14 stars), RO2 (2 stars), and LON (16 stars) candidate members of NGC 6253 (Table A.10). Figure 4 plots the CMDs with the positions of all the rotational variables within 
F. De Marchi et al.: Variable stars in NGC 6253
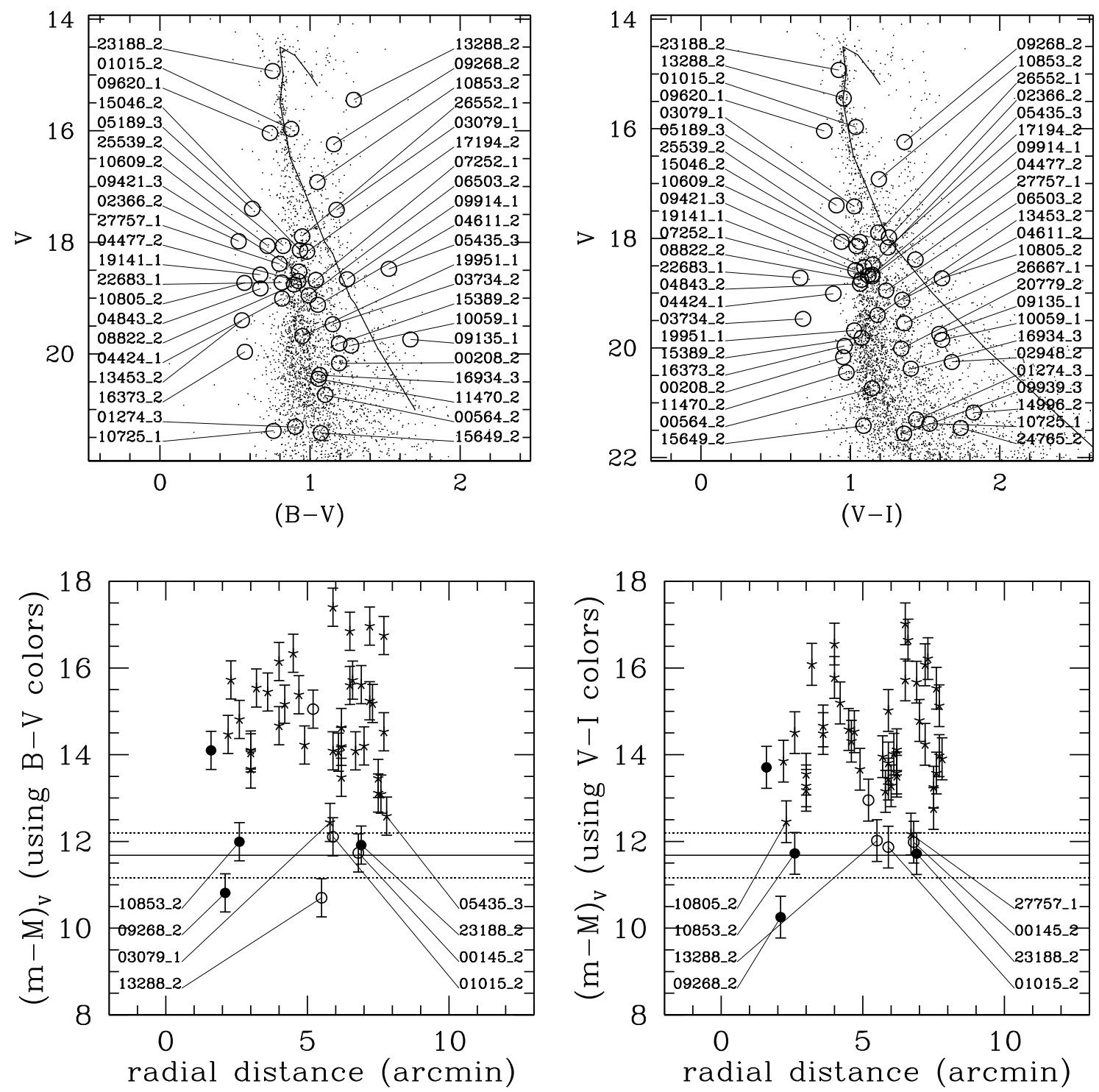

Fig. 3. Top row: colour-magnitude diagrams of NGC 6253 with the contact binaries at $r<8^{\prime}$ highlighted. The Main Sequences are individuated by fiducial lines. Bottom row: distance moduli of all contact binaries at $r<8^{\prime}$ obtained using the $P-L-C$ relations. We use both (B-V) (left panel) and $(V-I)$ colours (right panel). The horizontal line represents the distance modulus of the cluster resulting from isochrone fitting (Montalto et al. 2009). Filled circles show the binaries with MPs $>50 \%$; open circles the stars with MPs $<50 \%$, starred points the binaries with unknown membership. The error bars are the errors associated with the $M_{V}$ calculation and include errors in the colour determinations.

$8^{\prime}$ from the centre and the positions of the long-period variables highlighted (middle and right panels, respectively).

We discovered 27 variables in the $8^{\prime}$ radius from the centre and 16 of them can be considered candidate members on the basis of the positions on the CMDs and of the MPs (upper part of Table A.10). The stars 10042_2, 11077_2, and 06387_2 have a large MP and also a position on the CMDs compatible with cluster membership. We count 57 rotational variables in the surrounding field, i.e., an occurrence of $0.06 \mathrm{star}^{\operatorname{arcmin}^{-2}}$. This would imply an estimate of 12 field rotational variables along the line of sight of NGC 6253. There is a significant difference between the expected and the observed number of rotational variables, and we can infer that several selected candidate members (up to 15) actually belong to the cluster. Considering the short periods of these stars and the old age of the cluster, it is likely that the rotational variables that are cluster members are close, tidally locked binaries.
In the same manner, we can estimate $32 \mathrm{LON}$ field variables superposed to NGC 6253. In turn this means that up to 9 out of the $41 \mathrm{LON}$ variables discovered in the $8^{\prime}$ radius can be considered members of the cluster. These 9 stars should be found among the 16 candidate members listed in Table A.10.

\subsection{A new cataclysmic variable}

The U Geminorum variable 15877_2 is located at 6.8' from the cluster centre, but unfortunately its MP is not available (Table A.10). In the light curve, the scatter at the quiescence phase suggests some photospheric activity, but no periodicity is detected by analysing these measurements. Such a phase lastes the first 8 days of our survey. After that, its brightness in the $R$-band increases by about $2.5 \mathrm{mag}$ (Fig. 5). The maximum is not observed because it occurred in daytime. The star 15877_2 

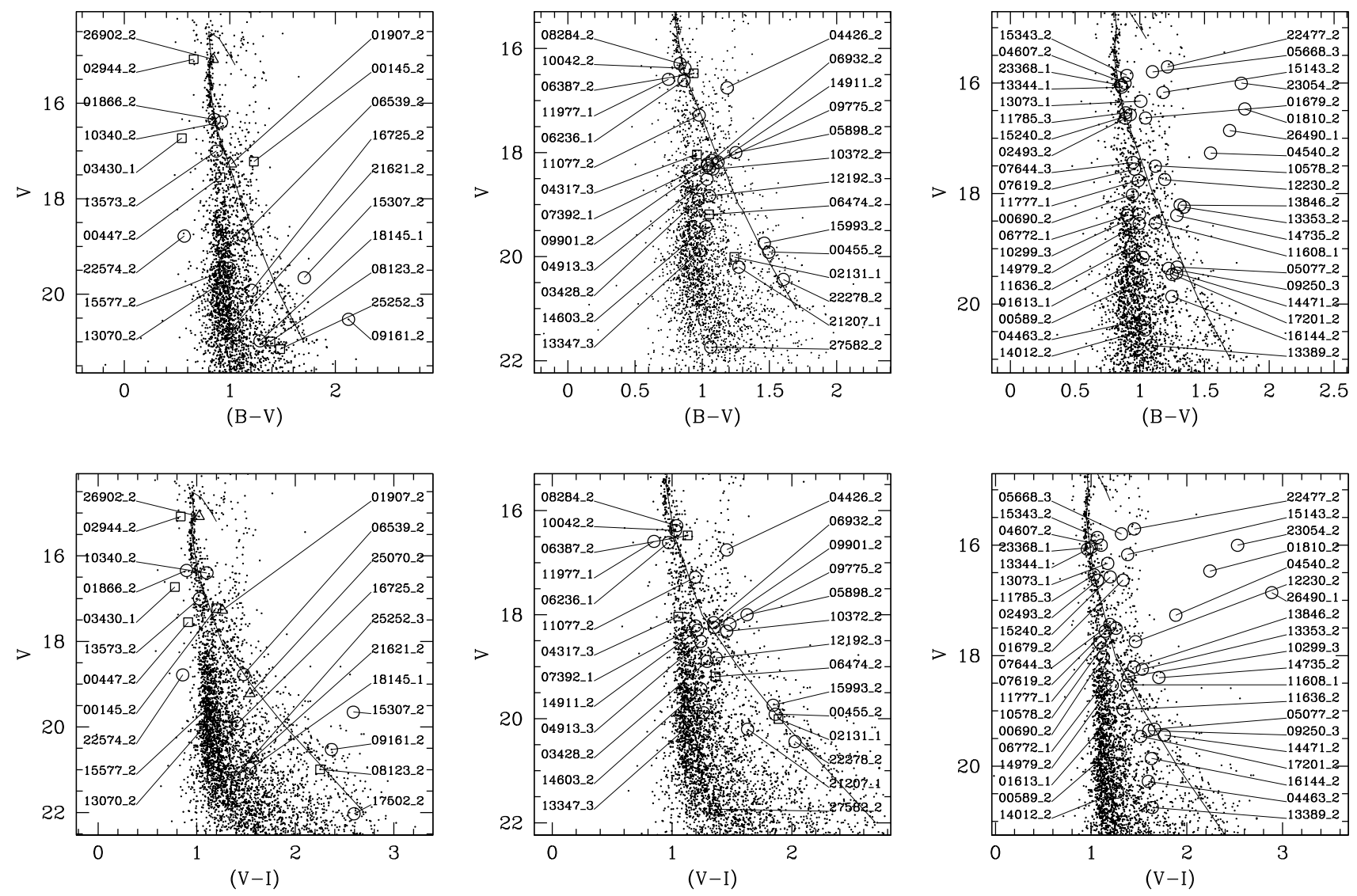

Fig. 4. Left panels: CMDs of NGC 6253 with the EA (circles), EB (squares), RS CVn (triangles) variables highlighted. Middle panels: CMDs of NGC 6253 with the rotational single-wave (circles), and double-wave (squares) variables highlighted. Right panels: CMDs of NGC 6253 with the long-period variables located within the $8^{\prime}$-radius circle highlighted. The Main Sequences are individuated by fiducial lines.

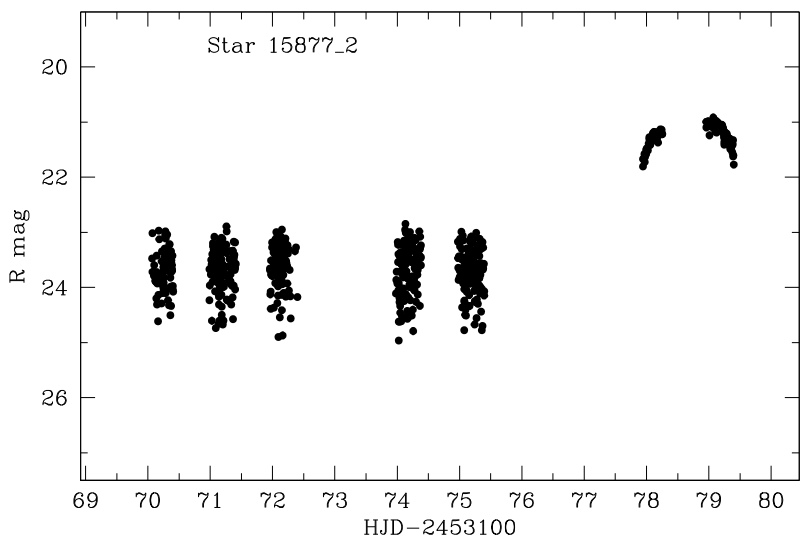

Fig. 5. Light curve of the new U Gem cataclysmic variable.

appears to be similar to the U Geminorum variable 06289_9, classified as a member of NGC 6791 (De Marchi et al. 2007).

\section{Conclusions}

In this paper we have described the first search for variable stars in the open cluster NGC 6253. Since the membership probabilities based on the proper motions are not reliable for stars with
$V>18$, only a few variables could be confirmed directly as cluster members. However, the comparison with the number of contact binaries and rotational variables (both short and long periods) found in a large area surrounding the cluster allowed us to estimate the incidence of these variables within the cluster, too. On the basis of these considerations we propose $35 \mathrm{mem}-$ bers of NGC 6253 within the sample of variable stars, though new observations are needed to identify some of them in an unambiguous way.

The class of main-sequence rotational variables is the most numerous, as observed in the surrounding field. On the basis of similar observing campaigns, we found the same number of contact binaries in NGC 6253 as were previously found in NGC 6791, thus confirming the anticorrelation between the frequency of binaries and the richness of the cluster (Kaluzny \& Rucinski 1995). This anticorrelation is similar to the one found between the frequency of blue stragglers and the total magnitude of the host cluster. Both these facts can lead back to the important effects caused by mass loss in the evolution and in the history of the dynamics of open clusters (Davies et al. 2004; De Marchi et al. 2006).

We discovered a new eruptive variable in NGC 6253. A single outburst was observed, so we cannot infer any physical characteristic of the system. Since we made the same discovery in NGC 6971 (De Marchi et al. 2007), it seems that continuous surveys on a few nights are very effective in finding these rare and interesting objects. 
Acknowledgements. This work was funded by COFIN 2004 "From stars to planets: accretion, disk evolution and planet formation" by MIUR and by PRIN 2006 "From disk to planetary systems: understanding the origin and demographics of solar and extrasolar planetary systems" by INAF. We thank the anonymous referee for careful reading and useful suggestions, and J. Vialle for checking the English form.

\section{References}

Alard, C. 2000, A\&A, 144, 363

Alard, C., \& Lupton, R. H. 1998, ApJ, 503, 325

Anthony-Twarog, B. J., Twarog, B. A., \& Mayer, L. 2007, AJ, 133, 1585

Bragaglia, A., Tessicini, G., Tosi, M., Marconi, G., \& Munari, U. 1997, MNRAS, 284, 477

Carpino, M., Milani, A., \& Nobili, A. M. 1987, A\&A, 181, 182

Carretta, E., Bragaglia, A., Tosi, M., \& Marconi, G. 2000, in Stellar Clusters and Associations: Convection, Rotation, and Dynamos, ed. R. Pallavicini, G. Micela, \& S. Sciortino, ASPC, 198, 273

Carretta, E., Bragaglia, A., \& Gratton, R. 2007, A\&A, 473, 129

Davies, M. B., Piotto, G., \& De Angeli, F. 2004, MNRAS, 349, 129

De Marchi, F. 2008, Ph.D. Thesis, University of Padova

De Marchi, F., De Angeli, F., Piotto, G., Carraro, G., \& Davies, M. B. 2006, A\&A, 459, 489

De Marchi, F., Poretti, E., Montalto, M., et al. 2007, A\&A, 471, 51

Kaluzny, J., \& Rucinski, S. M. 1995, A\&AS, 114, 1

Ferraz-Mello, S. 1981, AJ, 86, 4

Momany, Y., Vandame, B., Zaggia, S., et al. 2001, A\&A, 379, 436

Montalto, M., Piotto, G., Desidera, S., et al. 2007, A\&A, 470, 1137

Montalto, M., Piotto, G., Desidera, S., et al. 2009, A\&A, 505, 1129

Piatti, A. E., Clariá, J. J., Bica, E., Geisler, D., \& Minniti, D. 1998, AJ, 116, 801

Poretti, E. 2001, A\&A, 371, 986

Poretti, E., Clementini, G., Held, E., et al. 2008, ApJ, 685, 947

Rucinski, S. M. 2003, New Astron. Rev., 48, 703

Rucinski, S. M. 2007, MNRAS, 382, 393
Sagar, R., Munari, U., \& de Boer, K. S. 2001, MNRAS, 327, 23 Sestito, P., Randich, S., \& Bragaglia, A. 2007, A\&A, 465, 185 Stellingwerf, R. F. 1978, ApJ, 224, 953

Stetson, P. 1998, PASP, 106, 250

Tamuz, O., Mazeh, T., \& Zucker, S. 2005, MNRAS, 356, 1466

Twarog, B. R., Anthony-Twarog, B. J., \& De Lee, N. 2003, AJ, 125, 1383

Vaníček, P. 1971, Ap\&SS, 12, 10

Vasilevskis, S., Klemola, A., \& Preston, G. 1958, AJ, 63, 387

Weldrake, D. T. F., \& Bayliss, D. D. R. 2008, AJ, 135, 649

\section{Appendix A: Tables}

This Appendix includes the tables listing all the variables discovered in our survey of NGC 6253 and its surrounding field. The epochs of maximum or minimum brightness are expressed as HJD-2 453100 in the columns $T_{\max }$ and $T_{\min }$.

1. Pulsating variables: Table A.1;

2. EW-type variables: Table A.2;

3. EA-type variables: Table A.3;

4. EB-type variables: Table A.4;

5. RS-CVn variables: Table A.5;

6. rotational single-wave variables: Table A.6;

7. rotational double-wave variables: Table A.7;

8. long-period variables: Table A.8.

The binary systems considered as candidate members of NGC 6253 are listed in Table A.9. The rotational and longperiod variables considered as candidate members of NGC 6253 are listed in Table A.10.

Table A.1. Pulsating variables.

\begin{tabular}{|c|c|c|c|c|c|c|c|c|c|c|c|c|c|}
\hline Star & ID & Type & $\begin{array}{l}\alpha_{2000} \\
\text { [deg] }\end{array}$ & $\begin{array}{l}\delta_{2000} \\
\text { [deg] }\end{array}$ & $\begin{array}{c}\bar{R} \\
{[\mathrm{mag}]}\end{array}$ & $\begin{array}{c}\bar{V} \\
{[\mathrm{mag}]}\end{array}$ & $\begin{array}{l}\bar{B}-\bar{V} \\
{[\mathrm{mag}]}\end{array}$ & $\begin{array}{l}\bar{V}-\bar{I} \\
{[\mathrm{mag}]}\end{array}$ & $\begin{array}{c}T_{\max } \\
{[\mathrm{HJD}]}\end{array}$ & $\begin{array}{l}\text { Period } \\
{[\mathrm{d}]}\end{array}$ & $\begin{array}{c}\text { Ampl. } \\
{[R \text { mag] }}\end{array}$ & $\begin{array}{l}\text { Distance } \\
\text { [arcmin] }\end{array}$ & $\begin{array}{c}\mathrm{MP} \\
\%\end{array}$ \\
\hline $16334 \_6$ & 132171 & DSCT & 254.517933029 & -52.919478492 & 17.548 & 17.854 & 0.539 & 0.767 & 70.605 & 0.03065 & 0.02 & 15.6 & - \\
\hline $11353 \_4$ & 79692 & DSCT & 254.373216875 & -52.592925272 & 18.915 & 19.257 & 0.557 & 0.703 & 70.586 & 0.03818 & 0.04 & 16.0 & - \\
\hline 04756_6 & 123786 & DSCT & 254.547272025 & -53.053321129 & 16.815 & 17.179 & 0.568 & 0.762 & 70.586 & 0.04065 & 0.04 & 22.2 & - \\
\hline 22953_1 & 16369 & DSCT & 255.057077875 & -52.629821132 & 15.968 & 16.179 & 0.517 & 0.566 & 70.613 & 0.04182 & 0.02 & 11.4 & - \\
\hline 03523_3 & 48443 & DSCT & 254.462580529 & -52.719165053 & 15.640 & 15.871 & 0.501 & 0.562 & 70.582 & 0.04970 & 0.01 & 11.2 & - \\
\hline 16076_4 & 83210 & DSCT & 254.275776771 & -52.633207459 & 15.884 & 16.226 & 0.795 & 0.870 & 70.586 & 0.05494 & 0.06 & 18.6 & - \\
\hline 08420_6 & - & DSCT & 254.504434833 & -52.893020827 & 14.413 & 14.657 & 0.422 & 0.583 & 70.629 & 0.05611 & 0.02 & 14.7 & - \\
\hline 22047_5 & 112116 & DSCT & 254.376286383 & -53.047895213 & 17.184 & 17.491 & 0.543 & 0.669 & 70.586 & 0.05823 & 0.03 & 24.9 & - \\
\hline 04942_4 & 75001 & DSCT & 254.212624222 & -52.776630550 & 17.095 & 17.468 & 0.709 & 0.820 & 70.617 & 0.06244 & 0.04 & 20.7 & - \\
\hline 08514_6 & 126360 & DSCT & 254.438176757 & -52.890241485 & 17.543 & 17.897 & 0.626 & 0.839 & 70.590 & 0.06752 & 0.01 & 16.3 & - \\
\hline 08103_4 & 77295 & DSCT & 254.243943022 & -52.694411286 & 15.377 & 15.705 & 0.556 & 0.717 & 70.625 & 0.09455 & 0.01 & 9.2 & - \\
\hline 04796_8 & 168842 & ВCEP & 254.944203244 & -53.0415 & 19.135 & 19.163 & 0.084 & 0.223 & 70.602 & 0.0 & 0.05 & 21.0 & - \\
\hline 09747_6 & 127155 & HADS & 254.604170726 & -52.8303 & 16.301 & 16.582 & 0.463 & 0.672 & 70.586 & 0.06 & 0.09 & 9.5 & - \\
\hline 15098_6 & 131167 & HADS & 254.510582760 & -53.067284303 & 17.869 & 18.320 & 0.778 & 0.796 & 70.648 & 0.08412 & 0.09 & 23.5 & - \\
\hline 26414_6 & 139434 & HADS & 254.516592439 & -53.032263755 & 17.134 & 17.474 & 0.815 & 1.063 & 70.602 & 0.09374 & 0.43 & 21.5 & - \\
\hline 04116_6 & 123296 & HADS & 254.509177522 & -53.088555644 & 15.341 & 15.607 & 0.967 & 0.584 & 70.648 & 0.09771 & 0.24 & 24.7 & - \\
\hline 04180_4 & 74452 & RR & 254.326743597 & -52.796666245 & 15.052 & 15.283 & 0.541 & 0.542 & 70.594 & 0.31073 & 0.27 & 17.0 & - \\
\hline 10540_2 & 31353 & $\mathrm{RR}$ & 254.804741412 & -52.673375403 & 16.839 & 17.392 & 0.937 & 1.195 & 70.965 & 0.43030 & 0.20 & 2.4 & 72 \\
\hline 15578_7 & 157161 & $\mathrm{RR}$ & 254.658078793 & -52.867473297 & 15.698 & 16.416 & 1.104 & 1.057 & 71.051 & 0.51335 & 0.58 & 10.4 & - \\
\hline 03814_4 & 74180 & $\mathrm{RR}$ & 254.299365192 & -52.807157581 & 17.268 & 17.786 & 0.114 & 0.989 & 71.066 & 0.53648 & 0.68 & 18.1 & - \\
\hline
\end{tabular}


Table A.2. Contact binaries, W Ursae Maioris (EW) systems.

\begin{tabular}{|c|c|c|c|c|c|c|c|c|c|c|c|c|}
\hline Star & ID & $\begin{array}{l}\alpha_{2000} \\
\text { [deg] }\end{array}$ & $\begin{array}{l}\delta_{2000} \\
\text { [deg] }\end{array}$ & $\begin{array}{c}R_{\max } \\
{[\mathrm{mag}]}\end{array}$ & $\begin{array}{c}\bar{V} \\
{[\mathrm{mag}]}\end{array}$ & $\begin{array}{l}\bar{B}-\bar{V} \\
{[\mathrm{mag}]}\end{array}$ & $\begin{array}{c}\bar{V}-\bar{I} \\
{[\mathrm{mag}]}\end{array}$ & $\begin{array}{c}T_{\min } \\
\text { [HJD] }\end{array}$ & $\begin{array}{l}\text { Period } \\
\text { [d] }\end{array}$ & $\begin{array}{c}\text { Ampl. } \\
{[R \mathrm{mag}]}\end{array}$ & $\begin{array}{l}\text { Distance } \\
\text { [arcmin] }\end{array}$ & $\begin{array}{c}\mathrm{MP} \\
\%\end{array}$ \\
\hline 00441_4 & 71609 & 4.249749852 & 52.783032714 & 21.373 & 22.350 & - & - & 70.586 & 0.21002 & 0.38 & 19.5 & - \\
\hline 12192_1 & 8870 & 5.062764181 & -52.626856921 & 0.939 & 21.825 & 12 & 15 & 70.664 & 22407 & & 11.7 & - \\
\hline 10086_5 & 03181 & 4.190530241 & -52.847147618 & 21.454 & 2.485 & - & 1.488 & 70.766 & .22423 & & 22.6 & - \\
\hline 05190_4 & - & 4.295195930 & -52.770 & & - & - & - & 70.715 & & & 17.7 & - \\
\hline 16081_3 & 60 & & -52.6 & & 19.641 & 1.284 & 1.981 & & & & 8.1 & - \\
\hline $114 \_5$ & 96333 & 17 & 95 & & 20.725 & & 2.135 & & & & & - \\
\hline 02456_8 & 67381 & 5.061442816 & 52.915981403 & 99 & 1.376 & .135 & 1.700 & 70.773 & 53 & & 16.3 & - \\
\hline 6_2 & & 068 & 52.61 & & 1.178 & - & 1.824 & 609 & & & 6.0 & - \\
\hline $5 \_3$ & 63000 & 73 & 43 & & 20.850 & 1.521 & 2.258 & & & & 10.6 & - \\
\hline $27129 \_8$ & - & 38 & -52.993 & & 20.209 & .997 & 1.324 & & & & 18.9 & - \\
\hline 2710_1 & 16193 & 5.022664864 & -52.637298947 & 20.784 & 21.621 & 1.117 & 1.564 & 70.605 & 073 & .62 & 10.1 & - \\
\hline 1930_7 & 62711 & 4.784644788 & -52.843480211 & 18.230 & 19.355 & - & 1.605 & 70.633 & 4190 & 0.44 & 8.1 & - \\
\hline 17_5 & 06596 & 4.212008777 & -52.870254233 & .410 & 21.290 & 1.404 & 1.548 & & & & 22.5 & - \\
\hline 23722_8 & 182411 & 4.970157647 & -52.960 & & 0.371 & 0.908 & 1.344 & & & & 16.8 & - \\
\hline & 42656 & 9001 & -52.778 & & 21.460 & - & 1.739 & & & & 5.7 & - \\
\hline 26486_8 & 34282 & 143 & -52.931826300 & & 20.060 & 0.898 & 1.378 & & & & 15.5 & - \\
\hline & 30687 & & & & & - & 1.237 & & & & & - \\
\hline & 6 & & & & 60 & 1.346 & 1.371 & & & & & - \\
\hline & & & & & & & & & & & & - \\
\hline & 4 & & & & & & & & & & & - \\
\hline & 38 & & & & 13 & 4 & 1. & & & & & - \\
\hline & 22 & & & & .794 & 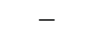 & 1.860 & & & & & - \\
\hline & 68 & & 1 & & 0.888 & .954 & 169 & & & & & - \\
\hline & 525 & & 24 & & .796 & .070 & 6 & & & & 4 & - \\
\hline & 829 & & 69 & & 20.013 & 0.897 & 1.079 & & & & .1 & - \\
\hline & 89 & & 00 & & 560 & - & .359 & & & & 7.6 & - \\
\hline & & & & & 897 & - & & & & & 7.2 & - \\
\hline & & 6 & 11 & & 713 & 412 & 25 & & & & & - \\
\hline & 172188 & 06 & -528 & 8 & 316 & 1.490 & 1.652 & & & & & - \\
\hline 26159_6 & 139249 & & 497 & & 19.514 & 0.887 & 1.421 & & & & & - \\
\hline & & & & & & & & & & & 2 & \\
\hline & & & & & & & & & & & .1 & 87 \\
\hline & 24811 & & & & 05 & 1.347 & & & & & & 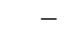 \\
\hline & 59 & & & & & - & & & & & & - \\
\hline & - & & & & - & - & - & & & & & \\
\hline & - & & & & .064 & 1 & 1.062 & & & & & \\
\hline & - & & & & 177 & 33 & 1.178 & & & & 7 & - \\
\hline 14 & 937 & & & & 21.638 & 30 & 2.200 & & & & 1 & \\
\hline & 44 & & & & 0.805 & 0.827 & 1.451 & & & & 8 & - \\
\hline & 53 & & & & 18.386 & 0.796 & 1.435 & & & & & - \\
\hline 4 & & & & & 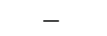 & - & - & & & & & - \\
\hline & & & & & & & & & & & & - \\
\hline 25579_5 & 14 & & 84 & & 67 & 11 & & & & & 6 & - \\
\hline & 698 & & & & 20.047 & & & & & & 3.8 & - \\
\hline & & & & & 22.010 & 0.497 & & & & & 5.1 & - \\
\hline 1_6 & 60 & & & & & - & & & & & 1.5 & - \\
\hline & & & & & & - & & & & & 4.6 & - \\
\hline & & & & & & & & & & & 3.0 & - \\
\hline & & & & & & - & & & & & & \\
\hline & & & & & & & & & & & & \\
\hline & & & & & & & & & & & & \\
\hline & & & & & & & & & & & & \\
\hline & & & & & & 99 & & & & & 17.3 & \\
\hline & & & & & & 0.902 & & & & & 7.7 & \\
\hline & 70496 & & & & & 0.484 & & & & & 17.5 & - \\
\hline & & & & & & 52 & & & & ( & 3.1 & $10^{*}$ \\
\hline & 79 & & -52 & & & 1.054 & & & & & 9.1 & \\
\hline 21127_7 & 162062 & & -53 . & 20.1 & 21.013 & - & 1.308 & & & & 9.4 & - \\
\hline $13285 \_5$ & - & 254. & -52.95 & & 21.889 & 1.920 & 1.165 & & & 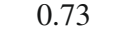 & 5.6 & - \\
\hline 16392_8 & 176839 & 255.070869741 & -53.089147343 & 19.690 & 20.420 & 1.317 & 1.475 & 70.766 & 8296 & 77 & 5.3 & - \\
\hline 14428_7 & 156121 & 5025 & -52 . & 20.591 & 20.920 & 0.680 & 0.586 & 70.594 & 00 & 7 & 10.8 & \\
\hline 26310_1 & 18660 & 255.00 & -52.7 & 20.568 & 21.468 & - & 1.411 & 70.637 & 0.28 & 0.21 & 9.5 & \\
\hline 15906_5 & 107493 & 254.316902665 & -53.047023021 & 19.467 & 20.264 & - & 1.244 & 70.605 & 0.28511 & 0.18 & 26.1 & \\
\hline
\end{tabular}


Table A.2. continued.

\begin{tabular}{|c|c|c|c|c|c|c|c|c|c|c|c|c|}
\hline Star & ID & $\begin{array}{l}\alpha_{2000} \\
{[\mathrm{deg}]}\end{array}$ & $\begin{array}{l}\delta_{2000} \\
{[\mathrm{deg}]}\end{array}$ & $\begin{array}{c}R_{\max } \\
{[\mathrm{mag}]}\end{array}$ & $\begin{array}{c}\bar{V} \\
{[\mathrm{mag}]}\end{array}$ & $\begin{array}{l}\bar{B}-\bar{V} \\
{[\mathrm{mag}]}\end{array}$ & $\begin{array}{c}\bar{V}-\bar{I} \\
{[\mathrm{mag}]}\end{array}$ & $\begin{array}{c}T_{\min } \\
{[\mathrm{HJD}]}\end{array}$ & $\begin{array}{c}\text { Period } \\
{[\mathrm{d}]}\end{array}$ & $\begin{array}{c}\text { Ampl. } \\
{[R \text { mag] }}\end{array}$ & $\begin{array}{l}\text { Distance } \\
\text { [arcmin] }\end{array}$ & $\begin{array}{c}\mathrm{MP} \\
\%\end{array}$ \\
\hline 09914_1 & 7211 & 54.964750204 & 52.662654779 & 17.972 & 18.661 & 1.247 & 1.147 & 70.582 & 0.28650 & 0.17 & 7.6 & - \\
\hline 00564_2 & 23439 & 4.689745025 & 52.812725870 & 20.204 & 0.735 & 1.101 & 1.145 & & 8755 & 0.07 & 6.9 & - \\
\hline 00920_4 & 71955 & 54.346375967 & 52.744068157 & 0.395 & 1.021 & 1.219 & 1.253 & 0.859 & .28946 & 0.20 & 15.6 & - \\
\hline 20677 & 62838 & 54.49250 & 52.71 & & .731 & 879 & 1.376 & & .28960 & 7 & 10.1 & - \\
\hline 17050_5 & 8433 & 45679 & 52.9 & & & 1.416 & 1.381 & & & & 24.8 & - \\
\hline 10327_5 & 5 & 54 & 9 & & & & 1.445 & & & & 18.6 & - \\
\hline 1788 & 7 & 3024 & 49 & 5 & 0.085 & 209 & 1.614 & & 0.29 & & 8.7 & - \\
\hline 08259_7 & 602 & 127 & 52.97 & 33 & .054 & 126 & 1.445 & & 59 & & 16.2 & - \\
\hline 108 & 31606 & 797 & 6128 & & 5.921 & .049 & 1.191 & 777 & 40 & & 2.6 & 78 \\
\hline 17587_5 & 108881 & 54.395 & 52.861 & 17.477 & 8.099 & 0.915 & 1.179 & & & & 16.4 & - \\
\hline 087 & 77784 & 7330 & 52.676 & 17.332 & 7.971 & - & 1.083 & 70.684 & 029 & & 19.6 & - \\
\hline t_4 & 77785 & 632 & -52.675 & 17.266 & 7.937 & - & 1.312 & 70.828 & 89 & & 19.6 & - \\
\hline 17194_2 & 36595 & 54.7504 & -52.583899859 & 17.339 & 8.167 & 0.980 & 1.251 & 0.742 & 0.29400 & & 7.5 & - \\
\hline 12169_5 & - & 880 & 18311 & 16.699 & - & - & - & & & & 24.0 & - \\
\hline 11470_2 & 32097 & 976 & -52.66 & 19.952 & 0.448 & 1.056 & 0.973 & & & & 3.2 & $21 *$ \\
\hline 10995_3 & 54399 & 142 & -52.57 & & & 1.202 & 1.085 & & & & 11.2 & - \\
\hline 13391_3 & 56428 & 190 & -52.7 & & & 1.212 & 1.035 & & & & 1.8 & - \\
\hline 079 & 170778 & 07 & -52 & & & 0.978 & 1.297 & & & & 16.2 & - \\
\hline & 14 & & 4 & & & & 1.074 & & & & & - \\
\hline & 3 & & 7 & & 15 & 1.010 & 1.109 & & & & & - \\
\hline 193 & 0504 & & 52. & & - & - & - & & & & 17.7 & - \\
\hline 107 & 7808 & & 52. & & 1.382 & .757 & 30 & & & & .9 & - \\
\hline 193 & 0505 & & 52. & & 2 & - & 1.599 & & & & 7.7 & - \\
\hline 19697_5 & 349 & 79 & 52.9 & & 38 & - & 1.467 & & & & 5.3 & - \\
\hline & 435 & 49 & 52.73 & 8 & 20 & 0.872 & 1.439 & & & & 6.5 & - \\
\hline 5 & 2507 & 136 & 52.96 & & 253 & 1.202 & 1.385 & & & & 24.8 & - \\
\hline & 402 & 32 & -52.9 & & 596 & .046 & 1.014 & & & & 7.5 & - \\
\hline & 434 & & -52.7 & & 617 & . & 111 & & & & 0.5 & - \\
\hline & & & 5 & & & 896 & & & & & & - \\
\hline 21824_4 & 765 & 592 & -52.7 & & & 0.853 & 1.042 & & & & 5.5 & - \\
\hline & & & -52 & & & 1.048 & & & & & 2 & - \\
\hline & & & 6 & & & - & & & & & .4 & - \\
\hline & & & & & & 1.187 & & & & & .4 & - \\
\hline & & & 0 & & & 1.088 & & & & & 19.8 & - \\
\hline & 22 & & 4 & & & - & 27 & & & & 8.3 & - \\
\hline & & & 4 & & & 0.887 & 84 & & & & 9.8 & - \\
\hline 5_4 & 83395 & & 52.6 & & & - & 1.744 & & & & 20.8 & - \\
\hline 12190_1 & 8868 & & 52.6 & & 2 & 1.189 & 1.317 & & & & 9.5 & - \\
\hline 163154 & 3394 & 82 & 52.62 & & 0 & - & 1.190 & 35 & & & 20.8 & - \\
\hline $51 \_1$ & 390 & & 52.7 & & 0 & .949 & 1.026 & & & & 4.2 & - \\
\hline $10 \_5$ & 30 & & -52 & & & - & 67 & & & & 9 & - \\
\hline $9 \_3$ & 658 & & 52. & & 3 & 619 & 69 & & & & 3 & - \\
\hline & 3488 & & & & & 12 & & & & & .3 & - \\
\hline 09421_3 & 054 & & 69 & & & 27 & 1.093 & & & & 5.9 & - \\
\hline $23060 \_4$ & - & & & & & 10 & & & & & 6.4 & - \\
\hline & & & & & & & & & & & 18.3 & - \\
\hline & & & & & & & & & & & 9.1 & - \\
\hline & & & & & & & & & & & 0 & - \\
\hline & & & & & & & & & & & & - \\
\hline & & & & & & & & & & & .0 & - \\
\hline & & & & & & 96 & 99 & & & & .2 & - \\
\hline & & & 2 & & & 1.037 & 78 & & & & 17.5 & - \\
\hline & & & 33 & & & - & 79 & & & & 5.9 & - \\
\hline 657 & 163128 & & 5 & & & 0 & 32 & & & & 10.5 & - \\
\hline & 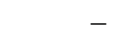 & & 500 & & & 6 & 1.133 & & & & 9.3 & - \\
\hline & 37 & & 50 & & & & 5 & & & & 7.5 & - \\
\hline 26855_6 & 139760 & 915 & -53.06 & 71 & 10 & 1.058 & 1.259 & 70.785 & & & 24.0 & - \\
\hline 27351_6 & 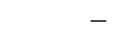 & 254 & -52.879 & 17.840 & 18.732 & 1.334 & 1.142 & 70.891 & & 0. & 15.2 & - \\
\hline 12869_5 & 105234 & 10159 & -52.971095051 & 18.995 & 19.687 & 0.865 & 1.146 & 70.602 & 2243 & 0.26 & 24.3 & - \\
\hline $24512 \_4$ & 578 & 845 & -52.555765616 & 18.176 & 18.880 & 0.894 & 1.426 & 70.812 & 315 & 0.36 & 18.2 & - \\
\hline 07369_6 & 125599 & 117 & -52.93 & 20.993 & 21.739 & - & 1.075 & 70.727 & & & 18.4 & - \\
\hline $18093 \_8$ & 178231 & 254 & -52 & 20 & 21.711 & & 1.621 & 70.738 & & & 14.2 & \\
\hline 22091_7 & - & 254.771066385 & -53.060847905 & 16.268 & 17.434 & 1.099 & 1.517 & 70.688 & 0.32533 & 0.41 & 21.2 & \\
\hline
\end{tabular}


Table A.2. continued.

\begin{tabular}{|c|c|c|c|c|c|c|c|c|c|c|c|c|}
\hline Star & ID & $\begin{array}{l}\alpha_{2000} \\
{[\mathrm{deg}]}\end{array}$ & $\begin{array}{l}\delta_{2000} \\
{[\mathrm{deg}]}\end{array}$ & $\begin{array}{c}R_{\max } \\
{[\mathrm{mag}]}\end{array}$ & $\begin{array}{c}\bar{V} \\
{[\mathrm{mag}]}\end{array}$ & $\begin{array}{l}\bar{B}-\bar{V} \\
{[\mathrm{mag}]}\end{array}$ & $\begin{array}{c}\bar{V}-\bar{I} \\
{[\mathrm{mag}]}\end{array}$ & $\begin{array}{c}T_{\min } \\
{[\mathrm{HJD}]}\end{array}$ & $\begin{array}{l}\text { Period } \\
\text { [d] }\end{array}$ & $\begin{array}{c}\text { Ampl. } \\
{[R \mathrm{mag}]}\end{array}$ & $\begin{array}{l}\text { Distance } \\
\text { [arcmin] }\end{array}$ & $\begin{array}{c}\text { MP } \\
\%\end{array}$ \\
\hline $22576 \_6$ & 136743 & $\overline{54.499200291}$ & -52.828093517 & 19.449 & 20.119 & 1.171 & 0.963 & 70.648 & 0.32801 & 0.26 & 12.2 & - \\
\hline & 182809 & 4.993242376 & -52.848196314 & 17.734 & 18.665 & .655 & 1.531 & 70.867 & 0.32875 & & 11.6 & - \\
\hline 12347_4 & 80409 & 4.338135370 & -52.562761802 & 18.107 & 19.099 & 16 & 1.694 & 70.688 & 3057 & & 18.0 & - \\
\hline 11806_5 & 104429 & 4.343834208 & -53.030153628 & 20.690 & 21.442 & 1.324 & 1.082 & 70.746 & .33164 & 0.25 & 24.7 & - \\
\hline 15757_3 & 58488 & 54.492893426 & -52.687986583 & 18.939 & 19.722 & 0.996 & 1.607 & 70.871 & .33170 & 0.22 & 10.2 & - \\
\hline 14706_7 & 56370 & 254.680160208 & -52.879691196 & 18.674 & 19.302 & 0.889 & 1.146 & 70.836 & .33554 & & 10.8 & - \\
\hline 22292_7 & - & 254.768480955 & -52.966907452 & & 17.741 & 0.876 & 1.107 & 70.906 & & & 15.5 & - \\
\hline 01904_7 & 14924 & 44 & & & 18.698 & 33 & 1.034 & & & & & - \\
\hline 11 & 5595 & 61 & 3 & & 19.711 & 0.779 & 1.123 & 70.637 & & & & - \\
\hline 07252_1 & 5281 & 193 & 52.704 & 17.917 & 18.677 & 1.038 & 1.120 & 70.684 & & & 1 & - \\
\hline 3_2 & 28181 & 962 & -52.728 & 271 & 18.952 & 0.992 & 1.239 & 70.816 & & & 2 & - \\
\hline 87 & 153458 & & 52.927 & & 19.292 & 1.180 & 1.044 & 70.828 & & & .1 & - \\
\hline 20307_3 & 62507 & 154 & -52.5708 & & 21.297 & - & 1.344 & 70.703 & & & 13.3 & - \\
\hline 27138_8 & 84766 & 5.089414723 & -52.990331243 & 18.288 & 18.980 & 1.010 & 1.337 & 70.738 & & 06 & 20.5 & - \\
\hline 05452_4 & 75369 & 4.336213743 & -52.762560934 & 726 & 20.477 & 0.965 & 1.122 & 70.664 & & & 16.1 & - \\
\hline & & 254.335 & -52.762955398 & & 20.620 & 0.897 & 1.146 & & & & & - \\
\hline & - & 254.540008213 & -52.645514980 & & 18.570 & 0.995 & 1.178 & & & & 9.2 & - \\
\hline 26137_6 & 39229 & 254.415882883 & -52.865801132 & 17.879 & 18.554 & 0.890 & 1.062 & 70.652 & & & 16.0 & - \\
\hline 17155_5 & 108526 & 622 & -52.910 & & 18.701 & 1.269 & & & & & & - \\
\hline 28186_6 & 0556 & 239 & & & 19.528 & 0.576 & 0.997 & & & & & - \\
\hline & 50 & & & & 20.975 & - & 1.482 & & & & & - \\
\hline & 277 & & & & 19.974 & 0.700 & 1.371 & & & & & - \\
\hline & - & & & & - & - & - & & & & & - \\
\hline & 54379 & & & & 9.640 & 630 & 1.328 & & & & & - \\
\hline 1 & 2271 & & & & 17.416 & 77 & 1.026 & & & & & - \\
\hline _3 3 & 54584 & & 44 & & 18.313 & 0.849 & 1.477 & & & & 6 & \\
\hline $3 \_2$ & 33652 & 95 & 27 & 96 & 19.397 & 0.546 & 1.182 & 70.773 & & ( & 5 & - \\
\hline & 9516 & 284 & 782 & 149 & 19.247 & 0.694 & 1.462 & 17 & & & 3 & - \\
\hline & & & & & & - & & & & & & - \\
\hline 86 & 128467 & 36 & -5300 & 35 & 20.199 & 0.964 & $1 .($ & & & & & - \\
\hline 09336_3 & 52979 & 849 & 499 & 17.533 & 18.111 & & 1.159 & & & & & - \\
\hline 924 & 77501 & 7595 & $-52.68 c$ & 134 & 18.750 & 0.943 & 1.128 & & & & & - \\
\hline & 947 & & & & 19.729 & & & & & & & - \\
\hline 00208_2 & 23152 & & & & 20.168 & & & & & & 3 & - \\
\hline & 19 & & & & 19.852 & 1.274 & & & & & 2 & - \\
\hline & 01 & & & & 882 & 0.777 & & & & & & - \\
\hline & & & & & 20.011 & - & 1.341 & & & & & - \\
\hline & & & & & - & - & - & & & & & \\
\hline & & & & & 18.828 & 0.669 & 1.065 & & & & & - \\
\hline 2 & & & & & 17.982 & 0.524 & 1.258 & & & & 2 & 35 \\
\hline 8_2 & 373 & & & & 19.231 & 0.973 & 0.772 & 46 & & & 3 & _- \\
\hline 8_3 & 391 & & 52.60 & & 19.601 & 1.048 & 1.195 & 82 & & ( & 10.1 & - \\
\hline & & & & & & 2 & & & & & & - \\
\hline 03734_2 & 25997 & 350 & -52.767 & & 19.469 & 1.151 & 0.683 & & & & 4.0 & - \\
\hline 09173_6 & 126796 & 626 & -52.8608 & 19.226 & 19.919 & 1.890 & 0.632 & & & & 1.6 & - \\
\hline 11723_4 & 958 & 3791 & -52.58 & & 20.458 & - & 1.017 & & & & 0.5 & - \\
\hline 11724_4 & 959 & & -52 & & 20.747 & - & 1.351 & & & & 0.5 & 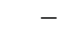 \\
\hline & & & & & 18.728 & 0.563 & & & & & 2.3 & $32 *$ \\
\hline & 849 & & & & & & & & & & 7.8 & - \\
\hline & - & & & & & & & & & & 29.3 & - \\
\hline & & & & & & & & & & & & \\
\hline & & & & & 60 & & & & & & 11.5 & \\
\hline & & & & & 61 & & & & & & & \\
\hline & & & & & 16.370 & 0. & & & & & 22.8 & - \\
\hline & & & & & 18.757 & 4 & & & & & 2.6 & $50 *$ \\
\hline & 66802 & & & & 20.072 & & & & & & 7.8 & 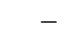 \\
\hline & 1571 & 24 & -52.85 & & 18.364 & & 0.677 & & & & 0.6 & - \\
\hline 20050_6 & 134857 & & -52.9 & & 18.352 & 0.828 & & & & & 16.5 & - \\
\hline 01015_2 & 23797 & 254.7 & -52.805 & 15.462 & 15.969 & 0.874 & 1.036 & 70.637 & & 0 & 5.9 & 22 \\
\hline 11185_6 & 128277 & 254.573301030 & -53.025133073 & 17.104 & 17.567 & 1.276 & 0.784 & 70.641 & & 0.27 & 0.3 & - \\
\hline 09984_6 & 127336 & 254.6 & -53.087164399 & 18.571 & 19.032 & 1.072 & 0.849 & 70.812 & & 0.25 & 23.4 & - \\
\hline 24135_2 & 42181 & 254.653419453 & -52.570100641 & 20.636 & 21.272 & 1.253 & 1.336 & 70.648 & 0.39393 & 0.14 & 9.3 & - \\
\hline 25832_5 & 115016 & 94018 & -52.9 & 15.564 & 16.213 & 0.963 & 0.867 & 70.777 & 60 & 0.28 & 24.6 & - \\
\hline 19024_2 & 38007 & 254.705000282 & -52.559692052 & 16.174 & 16.623 & 0.874 & 0.905 & 70.805 & 0.39520 & 0.12 & 9.2 & \\
\hline
\end{tabular}


Table A.2. continued.

\begin{tabular}{|c|c|c|c|c|c|c|c|c|c|c|c|c|}
\hline Star & ID & $\begin{array}{l}\alpha_{2000} \\
\text { [deg] }\end{array}$ & $\begin{array}{l}\delta_{2000} \\
{[\mathrm{deg}]}\end{array}$ & $\begin{array}{c}R_{\max } \\
{[\mathrm{mag}]}\end{array}$ & $\begin{array}{c}\bar{V} \\
{[\mathrm{mag}]}\end{array}$ & $\begin{array}{l}\bar{B}-\bar{V} \\
{[\mathrm{mag}]}\end{array}$ & $\begin{array}{c}\bar{V}-\bar{I} \\
{[\mathrm{mag}]}\end{array}$ & $\begin{array}{c}T_{\min } \\
{[\mathrm{HJD}]}\end{array}$ & $\begin{array}{l}\text { Period } \\
\text { [d] }\end{array}$ & $\begin{array}{c}\text { Ampl. } \\
{[R \text { mag] }}\end{array}$ & $\begin{array}{l}\text { Distance } \\
\text { [arcmin] }\end{array}$ & $\begin{array}{l}\text { MP } \\
\%\end{array}$ \\
\hline $22442 \_6$ & 36638 & 4.412274265 & $\overline{52.852975207}$ & 16.488 & 17.114 & 0.835 & 1.064 & 70.859 & 0.39560 & 0.17 & 15.6 & - \\
\hline 26667_1 & & 4.885399844 & 52.742269102 & & .550 & 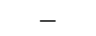 & 1.361 & & 39570 & & 4.6 & 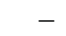 \\
\hline 097055 & 102913 & 2111 & 6286361 & & 411 & 989 & 1.028 & & 9580 & & 19.1 & - \\
\hline & & 563 & 1 & & 31 & 0.668 & 1.034 & & & & 4.7 & - \\
\hline & & & 58 & & 1 & 007 & 1.144 & & & & 13.1 & - \\
\hline 25 & 90082 & 6 & 8 & 9 & 8 & 11 & 19 & & & & 20.7 & - \\
\hline 5 & 6 & 9 & 52.8 & & 88 & 25 & 06 & & & & 18.9 & - \\
\hline 15 & 8 & 5 & $52 .($ & 2 & 42 & 33 & 42 & & & & 6.2 & $26^{*}$ \\
\hline 17 & 48 & & -52.9 & & 32 & 83 & 97 & & & & 17.8 & - \\
\hline 17494_8 & & 22 & -52.97 & 92 & 34 & 1.009 & 386 & & 41 & & 17.8 & - \\
\hline 229 & - & 79 & -52.87 & 17.804 & 130 & 49 & 283 & 82 & 08 & 0 & 10.3 & - \\
\hline & 85816 & 10 & -52.64 & 16.975 & 568 & .020 & 0.974 & & & & 19.8 & - \\
\hline & - & 18 & -52.8 & & 410 & 218 & 103 & & & & 17.9 & - \\
\hline & - & & 57 & & & & 07 & & & & 7.7 & 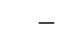 \\
\hline & 5585 & & -52 & & & & 0.880 & & & & 23.7 & - \\
\hline & & & -5 & & & & 1.207 & & & & .0 & - \\
\hline & & & & & & & & & & & 2.5 & - \\
\hline & 94 & & & & & 70 & 11 & & & & 16.7 & - \\
\hline & 3269 & & & & & & & & & & .5 & - \\
\hline & - & & 8 & & & 81 & 91 & & & & 14.8 & - \\
\hline & 521 & & 7 & & & 1.291 & 0.955 & & & & 5.5 & 3 \\
\hline & - & & -52 & & 4 & 0.947 & 1.186 & & & & 4.9 & 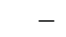 \\
\hline & - & & -52.5 & & 53 & 0.630 & 83 & & & & 9.6 & 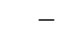 \\
\hline 2028 & 778 & & -52.92 & & 28 & 1.183 & 1.787 & & & & 20.7 & 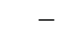 \\
\hline & 404 & & -52.61 & 64 & 28 & 0.751 & 0.923 & & & & 6.9 & 87 \\
\hline & 008 & & -52 & & 2 & 07 & 0.7 & & & & 14.3 & - \\
\hline & 175 & 99 & -5 & 7 & 1 & 0.812 & 0.665 & & & & 6.6 & 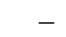 \\
\hline 6 & 9921 & 75 & -5 & 0 & 17.133 & 0.703 & 0.915 & & & & 13.4 & - \\
\hline & 128543 & 5 & -5 & & 8 & 1.695 & 2.151 & & & & 21.9 & - \\
\hline & 6991 & & -52.6 & 8 & 040 & 0.733 & 0.825 & 68 & & 0 & 6.2 & - \\
\hline 00188_1 & 146 & 835 & -52.81 & & 18.955 & 1.136 & 1.258 & & & & 11.0 & - \\
\hline 2166 & 87641 & 77 & -52.72 & 20.566 & 21.519 & 1.400 & 1.478 & & & & 16.5 & - \\
\hline 3 & 453 & 50 & -52 . & 16. & 16 & 1.016 & 1.010 & & & & 10.0 & \\
\hline 10609_2 & 31412 & 254.86241 & -52.6724 & 17.611 & 18.064 & 0.716 & 0.940 & & & & 4.0 & $58 *$ \\
\hline 15261_1 & 11141 & 255.068295962 & -52.576301385 & 17.344 & 17.955 & 0.896 & 1.286 & 70.695 & 0.8830 & 0.04 & 13.4 & \\
\hline
\end{tabular}


Table A.3. Detached systems, $\beta$ Per (Algol) type (EA) systems.

\begin{tabular}{|c|c|c|c|c|c|c|c|c|c|c|c|c|}
\hline Star & ID & $\begin{array}{l}\alpha_{2000} \\
{[\mathrm{deg}]}\end{array}$ & $\begin{array}{c}\delta_{2000} \\
{[\mathrm{deg}]}\end{array}$ & $\begin{array}{c}R_{\max } \\
{[\mathrm{mag}]}\end{array}$ & $\begin{array}{c}\bar{V} \\
{[\mathrm{mag}]}\end{array}$ & $\begin{array}{l}\bar{B}-\bar{V} \\
{[\mathrm{mag}]}\end{array}$ & $\begin{array}{c}\bar{V}-\bar{I} \\
{[\mathrm{mag}]}\end{array}$ & $\begin{array}{c}T_{\min } \\
{[\mathrm{HJD}]}\end{array}$ & $\begin{array}{c}\text { Period } \\
\text { [d] }\end{array}$ & $\begin{array}{c}\text { Ampl. } \\
{[R \text { mag] }}\end{array}$ & $\begin{array}{l}\text { Distance } \\
\text { [arcmin] }\end{array}$ & $\begin{array}{c}\mathrm{MP} \\
\%\end{array}$ \\
\hline 21471_1 & 15395 & 255.017261399 & -52.673015599 & 21.199 & - & - & - & 70.648 & 0.25051 & 0.86 & 9.2 & - \\
\hline 21839_2 & - & 254.800351011 & -52.691146980 & 22.995 & - & - & - & 70.598 & 0.25290 & 1.33 & 1.5 & - \\
\hline 256221 & 18191 & 255.016637408 & -52.559069751 & 20.972 & 21.863 & 1.266 & 1.657 & 70.621 & 0.26185 & 0.51 & 12.7 & - \\
\hline 060978 & 169599 & 254.909122586 & -52.998979068 & 20.318 & 21.536 & - & 1.888 & 70.844 & 0.27033 & 0.77 & 18.1 & - \\
\hline 00918_4 & 71954 & 254.224257122 & -52.743926019 & 20.481 & 21.772 & - & 2.829 & 70.734 & 0.28087 & 0.31 & 20.0 & - \\
\hline 10274_1 & 7474 & 255.048593809 & -52.656918798 & 17.406 & 17.994 & 0.990 & 1.223 & 70.695 & 0.31380 & 0.03 & 10.6 & - \\
\hline 19873_1 & 14344 & 255.075735833 & -52.720109603 & 21.198 & 22.435 & - & 2.534 & 70.625 & 0.31840 & 0.54 & 11.1 & - \\
\hline 02749_1 & 2022 & 254.997523924 & -52.776403366 & 20.313 & 21.331 & 1.269 & - & 70.914 & 0.34670 & 0.60 & 9.2 & - \\
\hline 21594_4 & 87592 & 254.326599726 & -52.724909290 & 18.588 & 19.615 & 1.102 & 1.840 & 70.961 & 0.38744 & 0.52 & 16.2 & - \\
\hline 26180_4 & 90782 & 254.247851409 & -52.796401894 & 20.325 & 21.216 & - & 1.701 & 70.801 & 0.39019 & 0.60 & 19.7 & - \\
\hline 23415 & 113232 & 254.331875018 & -53.089304114 & 20.261 & 21.598 & - & 3.018 & 70.637 & 0.39411 & 0.47 & 27.8 & - \\
\hline $16368 \_8$ & - & 254.941571694 & -53.093099122 & 18.420 & 19.074 & 1.008 & 1.387 & 70.707 & 0.41166 & 0.04 & 23.9 & - \\
\hline 15307_2 & 35129 & 254.830423589 & -52.609980387 & 18.574 & 19.654 & 1.706 & 2.588 & 70.707 & 0.44900 & 0.27 & 6.3 & $43 *$ \\
\hline 05088_8 & 169008 & 255.008837955 & -53.030705813 & 19.839 & 20.776 & 1.349 & 1.738 & 70.801 & 0.45707 & 0.32 & 21.2 & - \\
\hline 16525_8 & 176950 & 254.954548581 & -53.074149215 & 19.822 & 20.435 & 1.019 & 1.454 & 70.727 & 0.49625 & 0.29 & 22.9 & - \\
\hline 09161_2 & 30259 & 254.800143085 & -52.691547335 & 19.384 & 20.527 & 2.125 & 2.367 & 70.859 & 0.50611 & 0.69 & 1.5 & - \\
\hline 175022 & 36826 & 254.750395425 & -52.579915829 & 20.927 & 22.030 & - & 2.596 & 70.855 & 0.54690 & 0.44 & 7.7 & - \\
\hline 23787_1 & - & 255.0862 & -52.605776551 & 22.709 & - & - & - & 70.625 & 0.60500 & 0.91 & 13.0 & - \\
\hline 16725_2 & 36241 & 254.763 & -52.590 & 19.220 & 19.918 & 1.206 & 1.414 & 70.945 & 0.61 & 1 & 7.1 & $51 *$ \\
\hline 237 & 16924 & 255 & -52.60 & 54 & 20.717 & 1.329 & 1.495 & & 0.6 & 0 & 13.0 & - \\
\hline 04015_8 & 168422 & 255.08 & -53.07 & 20.996 & 22.309 & - & 2.539 & 70.734 & 0.6 & 0 & 24.6 & - \\
\hline 25809_8 & 183819 & 254.928 & -52.87 & 14.842 & 15.300 & 0.742 & 0.905 & 71.086 & 0.66 & 0 . & 11.4 & - \\
\hline 19258_2 & 38184 & 254.698073311 & -52.556586169 & 16.232 & 16.705 & 0.879 & 1.066 & 71.152 & 0.66468 & 0.01 & 9.5 & - \\
\hline 19192_2 & 38138 & 254.706610743 & -52.557441173 & 16.335 & 16.732 & 0.744 & 0.914 & 70.816 & 0.66606 & 0.01 & 9.4 & - \\
\hline 06539_2 & 28209 & 254.653146758 & -52.728429918 & 17.968 & 18.775 & 1.126 & 1.477 & 70.727 & 0.68230 & 0.19 & 4.4 & - \\
\hline 22574_2 & 40890 & 54.839652516 & -52.650662434 & 18.314 & 18.783 & 0.569 & 0.858 & 70.922 & 0.68170 & 0.51 & 4.3 & $0 *$ \\
\hline 23313_4 & 88780 & 254.275015010 & -52.619040328 & 18.097 & 18.925 & 1.176 & 1.453 & 71.031 & 0.69761 & 0.48 & 18.8 & - \\
\hline 28980_1 & 20459 & 255.057333782 & -52.738614108 & 20.906 & 21.667 & - & 1.391 & 70.891 & 0.73148 & 0.39 & 10.6 & - \\
\hline 01866_2 & 24487 & 254.653965066 & -52.793810375 & 15.769 & 16.343 & 0.853 & 0.897 & 70.883 & 0.85120 & 0. & 6.7 & 0 \\
\hline 11762_4 & 79983 & 254.318759486 & -52.581256371 & 21.279 & 22.243 & - & 1.450 & 70.926 & 0.86812 & 0.60 & 18.1 & - \\
\hline 04861_8 & 168880 & 254.993968428 & -53.039171477 & 15.044 & 15.559 & 0.736 & 0.864 & 70.746 & 0.90924 & 0.38 & 21.4 & - \\
\hline 2678488 & - & 255.081360466 & -53.089158714 & 18.167 & 18.618 & 0.844 & 0.954 & 71.309 & 0.90880 & 8 & 25.5 & - \\
\hline $26355 \_8$ & 184177 & 25500 & -52.9 & 31 & 21.496 & - & 2.555 & 168 & 0.9 & 6 & 20.8 & - \\
\hline $2 \_2$ & 42020 & 25 & -52 & 33 & 88 & 0.857 & 1.132 & & & & 8.0 & - \\
\hline 27820_8 & 185233 & 255 & -53.08 & 19.499 & 20.505 & - & 1.900 & 70.812 & 1.1 & 0. & 24.6 & - \\
\hline 07143_4 & 76597 & 254. & -52.71 & 14.554 & 15.003 & 0.910 & 0.861 & 71.289 & 1.2 & 0. & 14.6 & - \\
\hline 13070_2 & 33353 & 254.840405362 & -52.640163459 & 19.171 & 19.719 & 0.943 & 1.179 & 71.680 & 1.2424 & 0.30 & 4.8 & - \\
\hline 09324_4 & 78183 & 254.392031643 & -52.661470120 & 20.596 & 21.768 & - & 2.333 & 71.809 & 1.2979 & 0.18 & 14.1 & - \\
\hline 18145_1 & 13187 & 254.910993382 & -52.772099489 & 20.103 & 20.977 & 1.287 & 1.544 & 71.453 & 1.3306 & 0.3 & 6.4 & - \\
\hline 13414_8 & 174607 & 254.946603965 & -52.994282697 & 18.218 & 18.793 & 0.910 & 1.141 & 70.734 & 1.3531 & 0.1 & 18.3 & - \\
\hline 06441_8 & 169808 & 254.9060 & -52.988027454 & 21.352 & 21.832 & - & 1.531 & 71.828 & 1.4186 & 0. & 17.5 & - \\
\hline 09471_8 & 171895 & 254.964382916 & -52.896065953 & 14.733 & 15.260 & 0.845 & 1.047 & 71.703 & 1.4687 & 0.0 & 13.3 & - \\
\hline 25420_6 & 138746 & 254.432248297 & -53.067207957 & 17.993 & 18.753 & 1.284 & 1.385 & 71.047 & 1.5530 & 0.25 & 24.8 & - \\
\hline 08541_6 & 126376 & 254.518259628 & -52.889120371 & 14.441 & 14.919 & 0.810 & 0.964 & 71.004 & 1.5896 & 0.02 & 14.2 & - \\
\hline 20077_7 & 161149 & 254.813902734 & -52.906880213 & 18.541 & 19.413 & - & 0.603 & 70.738 & 1.6016 & 0.56 & 12.0 & - \\
\hline 03685_4 & 74090 & 254.340296069 & -52.810255408 & 17.827 & 18.464 & 0.986 & 1.184 & 71.129 & 1.6143 & 0.34 & 16.8 & - \\
\hline 04601_1 & 3387 & 254.986266354 & -52.747272966 & 18.870 & 19.686 & 1.316 & 1.522 & 71.828 & 1.6477 & 0.73 & 8.2 & - \\
\hline 27270_4 & 91435 & 254.240908904 & -52.603862690 & 19.017 & 19.833 & 1.040 & 1.344 & 71.449 & & 0.54 & 20.3 & - \\
\hline 19297_4 & 85797 & 254.393808310 & -52.651389139 & 17.894 & 18.420 & 0.749 & 0.999 & 72.008 & & 0.38 & 14.1 & - \\
\hline 09235_8 & 171720 & 255.015 & -52.902598679 & 20.206 & 20.899 & 1.114 & 1.243 & 72.266 & 1.7754 & 0.54 & 14.6 & - \\
\hline 10340_2 & 31195 & 254.706854237 & -52.676396811 & 15.894 & 16.397 & 0.920 & 1.103 & 72.051 & 1.8156 & 0.02 & 3.0 & 38 \\
\hline 18972_5 & 109822 & 254.280926335 & -53.016274254 & 17.332 & 18.047 & 0.926 & 1.132 & 71.656 & 1.8458 & 0.60 & 25.6 & - \\
\hline $16769 \_8$ & 177150 & 254.954720283 & -53.047084354 & 18.905 & 19.400 & 0.853 & 1.154 & 72.555 & 2.0715 & 0.3 & 21.4 & - \\
\hline $13573 \_2$ & 33743 & 254.81 & -52.633378426 & 16.518 & 17.003 & 0.873 & 1.027 & 71.039 & 2.3016 & 0.3 & 4.7 & 8 \\
\hline $25223 \_5$ & 114538 & 254.376854847 & -52.832836423 & 18.368 & 19.266 & 1.130 & 1.519 & 72.270 & 2.3134 & 0.28 & 16.1 & - \\
\hline $18853 \_2$ & 37870 & 254.821989999 & -52.561757998 & 20.125 & 21.214 & 1.470 & 2.068 & 72.613 & 2.6531 & 0.75 & 9.0 & - \\
\hline 19466_4 & 85930 & 254.205093525 & -52.634301683 & 18.845 & 19.616 & 1.088 & 1.379 & 71.688 & 2.6619 & 0.30 & 21.1 & - \\
\hline 27374_8 & 184932 & 254.953992006 & -52.870003666 & 18.837 & 19.562 & 1.094 & 1.438 & 72.004 & 3.7709 & 0.14 & 11.8 & - \\
\hline 15577_2 & 35339 & 254.696724869 & -52.606403320 & 18.945 & 19.457 & 0.994 & 1.155 & 72.207 & 3.7955 & 0.17 & 6.7 & - \\
\hline
\end{tabular}


Table A.4. Semi-detached systems, $\beta$ Lyr type (EB) systems.

\begin{tabular}{|c|c|c|c|c|c|c|c|c|c|c|c|c|}
\hline Star & ID & $\begin{array}{l}\alpha_{2000} \\
\text { [deg] }\end{array}$ & $\begin{array}{l}\delta_{2000} \\
{[\mathrm{deg}]}\end{array}$ & $\begin{array}{c}R_{\max } \\
{[\mathrm{mag}]}\end{array}$ & $\begin{array}{c}\bar{V} \\
{[\mathrm{mag}]}\end{array}$ & $\begin{array}{l}\bar{B}-\bar{V} \\
{[\mathrm{mag}]}\end{array}$ & $\begin{array}{l}\bar{V}-\bar{I} \\
{[\mathrm{mag}]}\end{array}$ & $\begin{array}{c}T_{\min } \\
{[\mathrm{HJD}]}\end{array}$ & $\begin{array}{l}\text { Period } \\
\text { [d] }\end{array}$ & $\begin{array}{c}\text { Ampl. } \\
{[R \text { mag] }}\end{array}$ & $\begin{array}{l}\text { Distance } \\
\text { [arcmin] }\end{array}$ & $\begin{array}{l}\text { MP } \\
\%\end{array}$ \\
\hline $00145 \_2$ & 23102 & 44.732762682 & -52.818849796 & 16.494 & 17.223 & 1.228 & 1.198 & 70.676 & 0.29110 & 0.26 & 6.8 & 0 \\
\hline 25252_3 & 36 & 610787192 & -52.641 & 20.3 & .152 & 1.477 & 1.395 & 67 & 80 & 7 & 7.1 & - \\
\hline 08123_2 & 437 & 265 & 364 & 4 & 2 & 35 & 252 & 33 & 03 & & 0.7 & - \\
\hline 268 & 9 & 96 & 237 & 66 & 505 & 7 & 061 & 36 & 28 & & 21.5 & - \\
\hline 46_3 & 57436 & 3 & 2.600 & 12 & 20.148 & 0 & 023 & 66 & 70 & & 9.9 & - \\
\hline 08031_6 & 032 & 4.470 & 52.9101 & 16.846 & 17.107 & 08 & 1.023 & 70.836 & 00 & 0 & 16.3 & - \\
\hline 00404_5 & 551 & 4.3408 & -53.063693904 & 17. & 18.366 & 0.523 & 1.202 & 70.602 & 41 & 0 & 26.4 & - \\
\hline 02944_2 & 375 & 081 & -52.778327877 & 14.636 & 15.080 & 0.658 & 0.837 & 70.766 & .42730 & 0.3 & 5.1 & 0 \\
\hline 22426_6 & 136623 & 30 & -52.857 & 19.344 & 20.014 & 1.182 & 1.383 & 70.711 & 50 & 0 & 12.8 & - \\
\hline 03930_4 & 263 & 4.37 & -52.80 & 15.918 & 16.494 & 0.743 & 0.943 & 70.781 & & & 15.3 & - \\
\hline t_7 & - & 07 & -52.84 & 18. & 71 & 55 & 0.916 & 70. & & & 9.0 & - \\
\hline & 55 & & 21 & & 61 & 0.716 & 1.133 & & & & 8.2 & - \\
\hline 11754_1 & 8551 & 255.0 & -52.63 & 16. & 55 & 0.708 & 0.603 & 70.785 & & & 10.0 & - \\
\hline 059 & - & & -53.00 & & & 0.785 & 0.904 & & & & 21.6 & - \\
\hline 27172_5 & 116076 & & -52.829020426 & & 17.402 & 0.963 & 1.112 & & & & 17.7 & - \\
\hline & & & -52.765747139 & & & 0.545 & 0.778 & & & & 7.2 & - \\
\hline & & & -52.55 & & 16.385 & 0.561 & 1.161 & & & & 0.0 & - \\
\hline & 4 & & -5 & & & 0.972 & 39 & & & & 15.6 & . \\
\hline 00447_2 & & & -52.8 & & & 0.910 & 0.913 & & & & 7.0 & - \\
\hline & 104162 & & & & & 0.786 & 0.913 & & & & 25.3 & . \\
\hline & 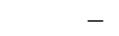 & & -52.89 & & & & & & & & 12.5 & - \\
\hline 15861_1 & 573 & 255.004 & -52.56 & 52 & 16.461 & 1.400 & 1.755 & 72.582 & 2.3596 & 0.05 & 12.0 & - \\
\hline
\end{tabular}

Table A.5. RS CVn variables, binary systems showing stellar activity in one or both components.

\begin{tabular}{|c|c|c|c|c|c|c|c|c|c|c|c|c|}
\hline Star & ID & $\begin{array}{l}\alpha_{2000} \\
{[\mathrm{deg}]}\end{array}$ & $\begin{array}{l}\delta_{2000} \\
{[\mathrm{deg}]}\end{array}$ & $\begin{array}{c}R_{\max } \\
{[\mathrm{mag}]}\end{array}$ & $\begin{array}{c}\bar{V} \\
{[\mathrm{mag}]}\end{array}$ & $\begin{array}{l}\bar{B}-\bar{V} \\
{[\mathrm{mag}]}\end{array}$ & $\begin{array}{c}\bar{V}-\bar{I} \\
{[\mathrm{mag}]}\end{array}$ & $\begin{array}{c}T_{\min } \\
{[\mathrm{HJD}]}\end{array}$ & $\begin{array}{l}\text { Period } \\
\text { [d] }\end{array}$ & $\begin{array}{c}\text { Ampl. } \\
{[R \text { mag] }}\end{array}$ & $\begin{array}{l}\text { Distance } \\
\text { [arcmin] }\end{array}$ & $\begin{array}{c}\mathrm{MP} \\
\%\end{array}$ \\
\hline 25516_5 & 114763 & 4.273572732 & 53.026533926 & 16.260 & 16.866 & 0.838 & 1.090 & 70.855 & - & 0.03 & 26.2 & - \\
\hline 02582_7 & 145527 & 4.864316020 & -53.055976199 & 18.6 & .343 & .072 & 1.358 & - & - & & 21.1 & - \\
\hline 20346_3 & 62538 & 4.569287024 & -52.559414721 & 19.0 & & & 1.399 & 70.8 & 0.4 & 0. & 11.6 & - \\
\hline 06395_3 & 50575 & 54.482527337 & -52591 & 17.001 & 7.755 & & 1.326 & & & & 12.6 & - \\
\hline 20136 & - & & & & & & & & & & 11.5 & _- \\
\hline $21621 \_2$ & & & & & & & & & & & 2.0 & - \\
\hline & & & & & & & & & & & .4 & - \\
\hline 05 & 86 & & 3 & & & & 7 & & & & .4 & - \\
\hline & 94 & & 0 & & & & 55 & & & & 21.5 & - \\
\hline 019 & 24521 & & 6 & & & 1.015 & 1.260 & & & & 5.5 & 0 \\
\hline & - & & 36 & & & - & 1.540 & & & & 2.4 & - \\
\hline 26281_4 & 90860 & 89 & -52.770 & 17 & 8.644 & 1.259 & 1.693 & 1.699 & & 0 & 16.4 & - \\
\hline 12708_1 & 9263 & 14 & -52.618 & 18. & 07 & 1.432 & 1.950 & 1.770 & & 0. & 12.8 & - \\
\hline 26902_2 & 079 & 0 & -52.73 & & 1 & 0.8 & 1.024 & 0 & & & 1.9 & 90 \\
\hline 5 & 107542 & - & -53 . & 1 & 27 & 1.091 & 1.362 & 11 & & & 28.1 & - \\
\hline 056 & 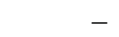 & 1 & -5 & 17.209 & 39 & $08>-1$ & 1.172 & & & & 1.4 & 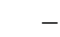 \\
\hline 129 & 65 & 0 & -5 & & 14.968 & 0.764 & 0.913 & 70. & 3. & $0 .($ & 2.7 & - \\
\hline 26602_8 & 184362 & 254.962161815 & -52.878228694 & 17.227 & 18.099 & 1.296 & 1.661 & 71.496 & 5.261 & 0.12 & 12.3 & - \\
\hline
\end{tabular}


Table A.6. Single-wave rotational variables (RO1).

\begin{tabular}{|c|c|c|c|c|c|c|c|c|c|c|c|c|}
\hline Star & ID & $\begin{array}{l}\alpha_{2000} \\
\text { [deg] }\end{array}$ & $\begin{array}{c}\delta_{2000} \\
\text { [deg] }\end{array}$ & $\begin{array}{c}\bar{R} \\
{[\mathrm{mag}]}\end{array}$ & $\begin{array}{c}\bar{V} \\
{[\mathrm{mag}]}\end{array}$ & $\begin{array}{l}\bar{B}-\bar{V} \\
{[\mathrm{mag}]}\end{array}$ & $\begin{array}{c}\bar{V}-\bar{I} \\
{[\mathrm{mag}]}\end{array}$ & $\begin{array}{c}T_{\min } \\
\text { [HJD] }\end{array}$ & $\begin{array}{l}\text { Period } \\
\text { [d] }\end{array}$ & $\begin{array}{c}\text { Ampl. } \\
{[R \mathrm{mag}]}\end{array}$ & $\begin{array}{l}\text { Distance } \\
\text { [arcmin] }\end{array}$ & $\begin{array}{c}\mathrm{MP} \\
\%\end{array}$ \\
\hline 02849_5 & 98253 & 254.354170197 & -52.871187225 & 14.559 & 15.082 & 0.792 & 0.989 & 70.805 & 0.34014 & 0.03 & 18.0 & - \\
\hline 08107_3 & 51940 & 4.473655174 & -52.562349360 & 18.548 & 19.122 & 908 & 132 & 70.648 & & & & - \\
\hline 26178_5 & 115311 & 4.352916128 & -52.832609795 & 19.503 & 19.605 & 304 & 0.474 & 70.723 & & & .9 & - \\
\hline 27582_2 & - & 4.7335 & -52.5 & 21.301 & 21.738 & .064 & 1.359 & & & & & - \\
\hline 07149_3 & 134 & 4.4793 & 68 & 17.269 & 17.713 & .766 & 0.958 & & 8 & & & - \\
\hline 0729_1 & 540 & 4.95391 & 52.8079 & 17.573 & 18.156 & .013 & .213 & 71.082 & & & 0 & - \\
\hline 5640_1 _ _ & 4131 & 55.021070972 & 52.7311 & 17.279 & 18.147 & .340 & .791 & 70.977 & .5781 & & & - \\
\hline 124 & 9051 & 708 & 52.622 & 18.647 & 19.365 & .246 & 1.473 & & & & & - \\
\hline 25160_6 & 38565 & 4.434291071 & -52.868736345 & 18.610 & 19.313 & .179 & 1.449 & 70.984 & 0.6806 & .05 & 15.5 & - \\
\hline 20185_4 & 86514 & 54.255951508 & -52.561648405 & 18.979 & 19.804 & 1.193 & 1.738 & 71.188 & 0.72130 & .14 & 20.7 & - \\
\hline 10203_3 & 53718 & 4.505967055 & -52.637261376 & 17.788 & 18.928 & 1.709 & 2.886 & 70.836 & .77319 & ר & 10.5 & - \\
\hline 22278_2 & 40654 & 54.649579305 & -52.665822056 & 19.457 & 20.440 & .604 & 2.026 & 70.988 & & & & - \\
\hline 06932_2 & 28498 & 770851 & -52.7224 & 17.550 & 18.151 & 1.072 & 1.347 & & & & & $10 *$ \\
\hline 06026_3 & 50300 & 254.537269269 & -52.6254 & 16.620 & 17.189 & 0.980 & 1.164 & & & & & 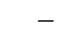 \\
\hline 49133 & 49473 & 254.567369728 & -52.73 & 17.894 & 18.513 & 1.035 & 1.240 & & & & & - \\
\hline $1 \_2$ & 30842 & 35 & -52 & 17.619 & 18.247 & 38 & & & & & & - \\
\hline 7_6 & 2 & & -52 & & 19.407 & & & & & & & - \\
\hline & 106 & & 3 & & & & & & & & & - \\
\hline 304 & 276 & & 63 & 17.007 & 17.576 & & 78 & & & & & - \\
\hline 302 & - & 7 & 52.6 & .032 & 17.626 & & 14 & & & & & - \\
\hline _1 & 217 & 35 & 08 & .367 & 20.202 & 73 & 31 & & & & & - \\
\hline 5_2 & 352 & 32 & 276 & .023 & 19.914 & 96 & 862 & & & & & - \\
\hline & 79141 & 09 & 701 & 16.422 & 16.938 & 0.841 & 1.017 & & & ( & 2 & - \\
\hline & 49977 & & 089 & 16.421 & 16.930 & 0.956 & 1.115 & & & ( & & - \\
\hline & & & 662 & .938 & 18.748 & .406 & 1.518 & & & & & - \\
\hline 02729_4 & 359 & 254. & -52.5 & 14.944 & 15.498 & 0.921 & 1.116 & & & & & - \\
\hline 13347_3 & 56388 & 989 & -52.7 & 19.284 & 19.884 & 0.984 & 1.263 & & & & & 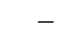 \\
\hline 14911_2 & 34814 & 254.7 & -52.6 & 17.702 & 18.299 & 1.053 & 1.212 & & & & & $63^{*}$ \\
\hline & & & & 17.212 & 18.001 & & & & & & & $53^{*}$ \\
\hline & 129324 & 01 & & 18.000 & 18.829 & 1.253 & & & & & & 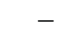 \\
\hline & & & & & & - & & & & & & - \\
\hline & & & & & & & & & & & & - \\
\hline & & & & & & & & & & & & \\
\hline & & & & 3 & 71 & & & & & & & 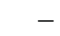 \\
\hline & 56571 & & 51 & 18 & 19.534 & & 3 & & & & & 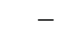 \\
\hline & 4548 & & 73 & .148 & 16.614 & 65 & 72 & & & & & _ \\
\hline & 8713 & & 52.63 & .185 & 16.592 & 0.749 & 9 & & & & & - \\
\hline $3 \_2$ & 4564 & & 52.6 & 860 & 19.425 & 29 & 39 & & & & & $43 *$ \\
\hline & & & 1 & 301 & 18.898 & 11 & 3 & & & & & $4 *$ \\
\hline & & & & & & & & & & & & $64 *$ \\
\hline 15421_8 & 176092 & 41 & -52.8 & 18.993 & 19.649 & 1.244 & 1.506 & & & & 11.1 & - \\
\hline 04426_2 & 545 & & 885 & 16.081 & 16.755 & 1.186 & 1.457 & & & & T.T. & 0 \\
\hline $17385 \_8$ & 177659 & & & 17.957 & 18.424 & & 94 & & & & 20.3 & 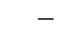 \\
\hline & 558 & & & & & & & & & & & - \\
\hline & - & & & & & & & & & & & - \\
\hline & & & & & & & & & & & & - \\
\hline & & & & & & & & & & & & \\
\hline & & & & & & & & & & & & \\
\hline & & & & & & & & & & & & \\
\hline & & & 27 & 720 & 19.604 & & 94 & & & c & 20.3 & - \\
\hline & 220 & & 10 & .650 & 18.317 & 30 & 6 & & & & & $26^{*}$ \\
\hline & 78029 & & 26 & & 3 & 2 & 0 & & & & & \\
\hline & 443 & & 70 & & 19.481 & & & & & & & - \\
\hline 8_4 & 80440 & & & & 17 & & & & & & & - \\
\hline 07392_1 & 5378 & 254.9 & -52.702 & 17.641 & 18.217 & 1.040 & 1.195 & 70.914 & & & 4.9 & 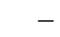 \\
\hline 10199_7 & 152324 & 271 & -52.944807129 & 16.754 & 17.555 & 1.244 & 1.546 & 70.738 & & 4 & 14.4 & 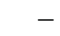 \\
\hline 10042_2 & 30960 & 254. & -52.67 & 15.946 & 16.379 & 0.872 & 1.029 & 445 & & 77 & 1.7 & 87 \\
\hline 13461_1 & 9832 & 254.962830875 & -52.606220233 & 15.034 & 15.459 & 0.791 & 0.886 & 71.523 & 3.100 & 0.01 & 9.3 & 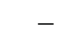 \\
\hline 15993_2 & 35676 & 438 & -52.600 & 18.851 & 19.741 & 1.462 & 1.844 & 71.766 & & 3 & 7.0 & \\
\hline 27870_8 & 185272 & 010 & -53.03 & 18.442 & 19.313 & - & 1.831 & & & & 21.6 & \\
\hline 11077_2 & 31786 & 254 & -52.66 & 16.725 & 17.281 & 0.978 & 1.194 & & 5.1 & 0. & & 1 \\
\hline 14568_1 & 10636 & 254.929608058 & -52.587730244 & 15.164 & 16.005 & 1.122 & 1.616 & 75.906 & - & 0.13 & 9.3 & \\
\hline
\end{tabular}


Table A.7. Double-wave rotational variables (RO2).

\begin{tabular}{crcccccccccrr}
\hline \hline Star & \multirow{2}{*}{ ID } & $\begin{array}{c}\alpha_{2000} \\
{[\mathrm{deg}]}\end{array}$ & $\begin{array}{c}\delta_{2000} \\
{[\mathrm{deg}]}\end{array}$ & $\begin{array}{c}\bar{R} \\
{[\mathrm{mag}]}\end{array}$ & $\begin{array}{c}\bar{V} \\
{[\mathrm{mag}]}\end{array}$ & $\begin{array}{c}\bar{B}-\bar{V} \\
{[\mathrm{mag}]}\end{array}$ & $\begin{array}{c}\bar{V}-\bar{I} \\
{[\mathrm{mag}]}\end{array}$ & $\begin{array}{c}T_{\min }[\mathrm{HJD}] \\
{\left[\begin{array}{c}\text { Period } \\
{[\mathrm{d}]}\end{array}\right.}\end{array}$ & $\begin{array}{c}\text { Ampl. } \\
{[R \text { mag }]}\end{array}$ & $\begin{array}{c}\text { Distance } \\
{[\mathrm{arcmin}]}\end{array}$ & $\begin{array}{c}\text { MP } \\
\%\end{array}$ \\
\hline 12912_3 & 56017 & 254.533555002 & -52.784475843 & 19.658 & 20.444 & 0.990 & 1.347 & 70.750 & 0.20639 & 0.07 & 9.8 & - \\
04317_3 & 49008 & 254.608573445 & -52.794792573 & 17.503 & 18.035 & 0.961 & 1.053 & 70.891 & 0.32733 & 0.03 & 7.8 & - \\
07671_4 & 76978 & 254.335531957 & -52.705889637 & 17.150 & 17.571 & 0.729 & 0.901 & 70.668 & 0.61286 & 0.03 & 15.8 & - \\
06474_2 & 28156 & 254.756128298 & -52.729271142 & 18.575 & 19.186 & 1.051 & 1.363 & 70.949 & 0.89900 & 0.06 & 1.4 & $9 *$ \\
06387_2 & 28088 & 254.793146373 & -52.730407627 & 15.970 & 16.476 & 0.937 & 1.130 & 71.191 & 0.92213 & 0.02 & 1.6 & 85 \\
14557_3 & 57443 & 254.447228258 & -52.600559305 & 19.027 & 20.148 & 1.753 & 2.378 & 70.828 & 0.95048 & 0.05 & 13.4 & - \\
05167_3 & 49661 & 254.490080023 & -52.710775679 & 16.300 & 16.647 & 0.626 & 0.746 & 70.637 & 1.03108 & 0.02 & 10.2 & - \\
26964_5 & 115910 & 254.232273105 & -52.879995622 & 15.669 & 16.302 & 0.884 & 1.200 & 71.410 & 1.22908 & 0.12 & 22.1 & - \\
02033_8 & 167096 & 254.957256487 & -52.945374763 & 15.467 & 15.955 & 0.670 & 1.060 & 70.906 & 1.590 & 0.09 & 15.7 & - \\
08864_1 & 6430 & 255.028469607 & -52.679450408 & 15.540 & 16.056 & 0.886 & 1.063 & 70.859 & 1.600 & 0.04 & 9.5 & - \\
02131_1 & 1588 & 254.946929133 & -52.786367467 & 19.053 & 20.007 & 1.239 & 1.889 & 71.059 & 1.8307 & 0.16 & 7.9 & - \\
14272_8 & 175212 & 255.031414981 & -52.944055117 & 16.372 & 16.886 & 0.859 & 1.033 & 72.906 & 2.4156 & 0.03 & 17.0 & - \\
09463_7 & 151658 & 254.785621657 & -52.955289040 & 14.720 & 15.184 & 0.806 & 0.938 & 72.711 & 2.7742 & 0.07 & 14.8 & - \\
19539_8 & 179361 & 255.012367131 & -53.058066501 & 14.733 & 15.184 & 0.730 & 0.913 & 70.793 & 3.204 & 0.04 & 22.7 & - \\
13211_7 & 155014 & 254.827269769 & -52.902388828 & 16.739 & 17.520 & 1.168 & 1.537 & 71.312 & 3.495 & 0.08 & 11.8 & - \\
\hline
\end{tabular}

Table A.8. Long-period variables.

\begin{tabular}{|c|c|c|c|c|c|c|c|c|c|c|}
\hline Star & ID & $\begin{array}{l}\alpha_{2000} \\
{[\mathrm{deg}]}\end{array}$ & $\begin{array}{l}\delta_{2000} \\
{[\mathrm{deg}]}\end{array}$ & $\begin{array}{c}\bar{R} \\
{[\mathrm{mag}]}\end{array}$ & $\begin{array}{c}\bar{V} \\
{[\mathrm{mag}]}\end{array}$ & $\begin{array}{l}\bar{B}-\bar{V} \\
{[\mathrm{mag}]}\end{array}$ & $\begin{array}{l}\bar{V}-\bar{I} \\
{[\mathrm{mag}]}\end{array}$ & $\begin{array}{c}\text { Ampl. } \\
{[R \text { mag] }}\end{array}$ & $\begin{array}{l}\text { Distance } \\
\text { [arcmin] }\end{array}$ & $\begin{array}{c}\mathrm{MP} \\
\%\end{array}$ \\
\hline 15877_2 & - & 254.703470476 & -52.602380112 & 23.035 & - & - & - & $3.0:$ & 6.8 & - \\
\hline 11351_8 & 173273 & 254.896720660 & -52.836752470 & 14.465 & 15.205 & 1.285 & 1.523 & 0.07 & 9.0 & - \\
\hline 26313_3 & 67881 & 54.415548223 & -52.753401371 & 13.999 & 15.261 & 1.798 & 2.677 & 0.04 & 13.2 & - \\
\hline 05327_6 & 124207 & 254.603157998 & -53.029501689 & 14.850 & 15.324 & 0.881 & 0.957 & 0.03 & 20.2 & - \\
\hline 04281_3 & 48982 & 254.572575855 & -52.797861044 & 14.531 & 15.331 & 1.279 & 1.495 & 0.02 & 9.0 & - \\
\hline 04319_3 & 49009 & 254.427392028 & -52.794585896 & 14.588 & 15.372 & 1.254 & 1.491 & 0.03 & 13.5 & - \\
\hline 23474_5 & 113269 & 254.198230796 & -53.082982900 & 14.599 & 15.400 & 1.471 & 1.531 & 0.09 & 30.6 & - \\
\hline 03878_4 & 74227 & 254.188239396 & -52.805213798 & 14.630 & 15.417 & 1.169 & 1.485 & 0.05 & 21.9 & - \\
\hline 16505_4 & - & 254.277901396 & -52.611645452 & 14.891 & 15.439 & 0.856 & 1.077 & 0.02 & 18.9 & - \\
\hline 13952_7 & 55685 & 54.833974119 & -52.891196229 & 14.937 & 15.508 & 1.005 & 1.127 & 0.04 & 11.2 & - \\
\hline 05891_1 & 4306 & 255.080076806 & -52.726794719 & 14.989 & 15.633 & 0.969 & 1.202 & 0.02 & 11.3 & - \\
\hline 25606_6 & 138852 & 254.495945379 & -53.020895006 & 15.119 & 15.634 & 0.904 & 1.018 & 001 & 21.2 & - \\
\hline 22477_2 & 40819 & 254.690203633 & -52.656152556 & 15.014 & 15.708 & 1.214 & 1.451 & 0 & 4.3 & 3 \\
\hline 01236_4 & 72177 & 254.1 & -52.713 & 15.135 & 15.725 & 0.933 & 1.111 & $0 .($ & 21.2 & - \\
\hline 05668_3 & 50025 & $\$ 26$ & -52.66 & 38 & 15.796 & 1.098 & 1.317 & & 6.0 & - \\
\hline 10184_1 & 7408 & 60 & -52.658 & 14.729 & 15.809 & 1.755 & 2.343 & & 2.1 & - \\
\hline 04173_6 & 123343 & 54.62 & -53.085 & 15.328 & 15.833 & 0.881 & 1.001 & 0.0 & 23.2 & - \\
\hline 15343_2 & - & 254.77 & -52.609 & 15.370 & 15.863 & 0.900 & 1.064 & 0.0 & 5.9 & - \\
\hline 04540_6 & 123621 & 254.485832906 & -53.064431642 & 15.397 & 15.904 & 0.881 & 0.996 & 0.02 & 23.7 & - \\
\hline 23883_4 & 89157 & 254.216074468 & -52.590141284 & 15.356 & 15.925 & 0.937 & 1.108 & 0.0 & 21.4 & - \\
\hline 08512_7 & 150821 & 54.695827862 & -52.969171258 & 15.402 & 15.957 & 0.883 & 1.104 & 0.0 & 15.9 & - \\
\hline 17106_4 & 84006 & 54.286044592 & -52.582957236 & 15.454 & 15.967 & 0.884 & 1.025 & 0.0 & 19.2 & - \\
\hline 14599_1 & 10660 & 54.934027456 & -52.587221603 & 15.334 & 15.975 & 1.062 & 1.220 & $0 .($ & 9.4 & - \\
\hline 04607_2 & 26693 & 54.776657557 & -52.755229164 & 15.494 & 16.002 & 0.886 & 1.105 & 0. & 2.8 & 0 \\
\hline 23054_2 & 41297 & 54.729 & -52.6232 & 14.916 & 16.005 & 1.784 & 2.530 & 0. & 5.3 & 12 \\
\hline 23368_1 & 16649 & 254.892499238 & -52.618200382 & 15.549 & 16.040 & 0.856 & 1.000 & 0 . & 7.0 & - \\
\hline 19050_8 & 178988 & 254.931003357 & -53.083061626 & 15.481 & 16.064 & 0.921 & 1.148 & 0. & 23.2 & - \\
\hline 13344_1 & 9747 & 254. & -52.6 & 15.602 & 16.076 & 0.860 & 0.961 & & 7.2 & - \\
\hline 247 & 114148 & 25 & -52.9 & 45 & 098 & 0.851 & 1.026 & & 22.7 & - \\
\hline 2 & 3 & 1 & -52.6 & 30 & 16.174 & 1.180 & 1.382 & 0.01 & 6.1 & 58 \\
\hline 13431_1 & 9810 & 550 & -52.60 & 15.655 & 16.205 & 0.924 & 1.080 & 0 & 13.1 & - \\
\hline 17430_5 & 108741 & 254.33 & -52.880 & 14.843 & 16.279 & 1.754 & 3.260 & 0 & 19.0 & - \\
\hline 13073_1 & 9538 & 54.881018074 & -52.612942861 & 15.707 & 16.331 & 1.007 & 1.173 & 0.02 & 7.0 & - \\
\hline 11792_8 & 173579 & 54.951230554 & -53.084956409 & 15.516 & 16.349 & 1.325 & 1.605 & 0.0 & 23.5 & - \\
\hline 08457_7 & 150775 & 254.722277418 & -52.969935325 & 15.796 & 16.369 & 0.864 & 1.120 & $0 .($ & 15.8 & - \\
\hline $12453 \_8$ & 173994 & 254.912800088 & -53.049048629 & 15.765 & 16.391 & 1.009 & 1.252 & $0 .($ & 21.1 & - \\
\hline 26211_8 & 184067 & 54.901 & -53.057270437 & 15.784 & 16.419 & 1.014 & 1.239 & $0 .($ & 21.5 & - \\
\hline 23737_4 & 89067 & 254.236802502 & -52.598492046 & 15.794 & 16.432 & 0.975 & 1.217 & $0 .($ & 20.5 & - \\
\hline 05982_3 & 50264 & 254.421725658 & -52.629770606 & 15.096 & 16.452 & 1.995 & 3.172 & 0.07 & 13.6 & - \\
\hline 01810_2 & 24442 & 254.684592868 & -52.794551835 & 15.350 & 16.469 & 1.811 & 2.241 & 0.01 & 6.0 & - \\
\hline 28407_4 & 92169 & 254.336300773 & -52.563553968 & 15.807 & 16.470 & 1.016 & 1.239 & 0.01 & 18.0 & - \\
\hline 02433_7 & 145390 & 254.801403096 & -53.058306375 & 15.823 & 16.507 & 1.064 & 1.298 & 0.03 & 21.0 & - \\
\hline 11785_3 & 55053 & 254.627601281 & -52.719312463 & 16.004 & 16.539 & 0.892 & 1.031 & $0 .($ & 5.2 & - \\
\hline 17464_5 & 108772 & 254.184033572 & -52.876729556 & 15.712 & 16.558 & 1.155 & 1.479 & 0.05 & 23.6 & - \\
\hline
\end{tabular}


Table A.8. continued.

\begin{tabular}{|c|c|c|c|c|c|c|c|c|c|c|}
\hline Star & ID & $\begin{array}{l}\alpha_{2000} \\
\text { [deg] }\end{array}$ & $\begin{array}{c}\delta_{2000} \\
{[\mathrm{deg}]}\end{array}$ & $\begin{array}{c}\bar{R} \\
{[\mathrm{mag}]}\end{array}$ & $\begin{array}{c}\bar{V} \\
{[\mathrm{mag}]}\end{array}$ & $\begin{array}{l}\bar{B}-\bar{V} \\
{[\mathrm{mag}]}\end{array}$ & $\begin{array}{l}\bar{V}-\bar{I} \\
{[\mathrm{mag}]}\end{array}$ & $\begin{array}{c}\text { Ampl. } \\
{[R \text { mag] }}\end{array}$ & $\begin{array}{l}\text { Distance } \\
\text { [arcmin] }\end{array}$ & $\begin{array}{c}\mathrm{MP} \\
\%\end{array}$ \\
\hline 16742_5 & 108164 & 254.238860400 & -52.956317691 & 15.582 & 16.560 & 1.591 & 1.823 & 0.02 & 24.4 & - \\
\hline 15240_2 & 35074 & 254.740026868 & -52.611151405 & 16.008 & 16.576 & 0.926 & 1.199 & 0.05 & 5.9 & 6 \\
\hline 22227_5 & - & 254.382144163 & -53.009810280 & 15.924 & 16.609 & 0.977 & 1.329 & 0.05 & 22.9 & - \\
\hline 04392_8 & 168622 & 254.957143445 & -53.056818971 & 14.741 & 16.622 & 2.001 & 4.657 & 0.12 & 22.0 & - \\
\hline 14798_7 & 156453 & 254.850526253 & -52.878232640 & 15.768 & 16.633 & 1.230 & 1.547 & 0.01 & 10.6 & - \\
\hline 01679_2 & 24338 & 254.824880791 & -52.795983855 & 15.967 & 16.634 & 1.044 & 1.334 & 0.04 & 5.6 & - \\
\hline 02493_2 & 25002 & 254.669614398 & -52.785376915 & 16.149 & 16.636 & 0.888 & 1.066 & 0.02 & 5.9 & 0 \\
\hline $11105 \_6$ & 128214 & 254.581143422 & -53.029053684 & 16.198 & 16.735 & 0.910 & 1.065 & 0.01 & 20.4 & - \\
\hline $14222 \_8$ & 175175 & 254.929878098 & -52.947505054 & 16.240 & 16.803 & 0.947 & 1.099 & 0.02 & 15.5 & - \\
\hline 02875_7 & 145798 & 254.655457746 & -53.052033581 & 16.259 & 16.849 & 0.925 & 1.154 & 0.02 & 21.0 & - \\
\hline 26490_1 & 18773 & 254.913679865 & -52.759462661 & 15.645 & 16.860 & 1.694 & 2.887 & 0.01 & 6.0 & - \\
\hline 26475_5 & 115537 & 254.183141777 & -53.019781837 & 16.256 & 16.862 & 0.905 & 1.098 & 0.02 & 28.3 & - \\
\hline 20138_5 & 110664 & 254.187023915 & -52.933300785 & 15.228 & 16.870 & 1.965 & 3.971 & 0.06 & 25.1 & - \\
\hline 19794_7 & 160897 & 254.832980735 & -52.940633495 & 16.073 & 16.887 & 1.108 & 1.580 & 0.02 & 14.1 & - \\
\hline 04395_3 & 49073 & 254.578820499 & -52.787020479 & 16.047 & 16.917 & 1.300 & 1.625 & 0.03 & 8.4 & - \\
\hline 13982_1 & 10216 & 254.980745814 & -52.597526691 & 16.360 & 16.968 & 1.040 & 1.176 & 0.01 & 10.1 & - \\
\hline 15500_6 & 131491 & 254.428654489 & -53.016789930 & 16.375 & 16.997 & 1.242 & 1.318 & 0.09 & 22.3 & - \\
\hline 28039_6 & 140502 & 254.609979317 & -52.873147902 & 16.434 & 17.038 & 0.974 & 1.176 & 0.01 & 11.5 & - \\
\hline 13872_8 & 174918 & 255.076783646 & -52.967649449 & 16.294 & 17.068 & 1.099 & 1.494 & 0.06 & 19.1 & - \\
\hline 24621_6 & 138144 & 254.616811813 & -53.040489156 & 16.527 & 17.125 & 0.943 & 1.161 & 0.02 & 20.7 & - \\
\hline 23516_4 & 88917 & 254.180 & -52.608 & 16.534 & 17.137 & 0.985 & 1.194 & 01 & 22.3 & - \\
\hline 24991_5 & 114351 & 254.384331589 & -52.864 & 16.519 & 17.162 & 0.968 & 1.171 & .02 & 16.9 & - \\
\hline 11266_7 & 153286 & 54.8650 & -52.929625368 & 16.652 & 17.173 & 0.869 & 1.035 & 0.02 & 13.7 & - \\
\hline 00503_1 & 371 & 255.087393181 & -52.811569453 & 16.474 & 17.183 & 1.168 & 1.408 & 0.03 & 13.1 & - \\
\hline 10707_1 & 7796 & 255.033486335 & -52.650170323 & 16.648 & 17.203 & 0.924 & 1.124 & 0.02 & 10.2 & - \\
\hline $13425 \_7$ & 155210 & 254.840832640 & -52.899288938 & 16.681 & 17.219 & 0.831 & 1.077 & 0.05 & 11.7 & - \\
\hline 10573_7 & 152651 & 254.796253882 & -52.939824135 & 16.472 & 17.221 & 1.105 & 1.374 & 0.02 & 13.9 & - \\
\hline 04428_3 & 49099 & 254.432226579 & -52.783712525 & 16.715 & 17.237 & 0.849 & 1.070 & 0.01 & 13.1 & - \\
\hline 04646_4 & 74783 & 254.320409138 & -52.784648121 & 15.544 & 17.265 & 1.971 & 4.171 & 007 & 17.0 & - \\
\hline 04540_2 & 26638 & 254.854179209 & -52.755971227 & 16.316 & 17.268 & 1.547 & 1.884 & 0.01 & 4.2 & 0 \\
\hline 27239_6 & 140023 & 254.438576590 & -52.970843095 & 16.726 & 17.370 & 0.996 & 1.227 & 0.03 & 19.8 & - \\
\hline 25810_5 & 115001 & 254.384646499 & -52.948918666 & 16.551 & 17.383 & 1.132 & 1.508 & 0.03 & 20.1 & - \\
\hline 19606_6 & 134536 & 254.621391134 & -52.935510530 & 16.703 & 17.415 & 1.173 & 1.419 & 0.08 & 14.7 & - \\
\hline 17003_7 & 158447 & 254.811951103 & -52.847685721 & 16.681 & 17.418 & 1.143 & 1.425 & .04 & 8.5 & - \\
\hline 07644_3 & 51551 & 254.60 & -52.64 & 16.818 & 17.437 & 0.947 & 1.196 & 2 & 7.2 & - \\
\hline 05697_7 & 148296 & 83 & -53.00 & 16.702 & 17.438 & 1.164 & 1.444 & 0.03 & 18.1 & - \\
\hline 30092_1 & - & 255.090 & -52.670 & 16.835 & 17.456 & 1.065 & 1.276 & 0.0 & 11.9 & - \\
\hline 10578_2 & 31384 & 254.734 & -52.673090562 & 16.890 & 17.505 & 1.120 & 1.259 & 0.0 & 2.5 & - \\
\hline 15276_8 & 175977 & 255.032763976 & -52.885173750 & 16.830 & 17.535 & 1.125 & 1.439 & 0.03 & 14.2 & - \\
\hline 07619_2 & 29043 & 254.811684961 & -52.712570710 & 17.009 & 17.559 & 0.960 & 1.147 & 0.02 & 1.5 & 7 \\
\hline 18827_4 & 85412 & 254.306270509 & -52.702248593 & 16.915 & 17.624 & 1.148 & 1.337 & 0.02 & 16.9 & - \\
\hline 15065_6 & 131140 & 254.478160687 & -53.072171326 & 17.010 & 17.658 & 1.070 & 1.307 & 0.03 & 24.3 & - \\
\hline 00879_1 & 650 & 255.016171891 & -52.805376567 & 16.955 & 17.668 & 1.158 & 1.380 & 0.03 & 10.6 & - \\
\hline 09335_3 & 52978 & 254.443067690 & -52.713365695 & 16.956 & 17.709 & 1.428 & 1.576 & 0.04 & 11.9 & - \\
\hline 12313_3 & 55498 & 254.612422276 & -52.606934830 & 16.891 & 17.727 & 1.285 & 1.606 & 0.03 & 8.4 & - \\
\hline 03467_7 & 146313 & 254.720012598 & -53.043004564 & 16.823 & 17.731 & 1.135 & 1.767 & 0.09 & 20.2 & - \\
\hline 12230_2 & 32699 & 254.815958044 & -52.651011235 & 17.029 & 17.748 & 1.192 & 1.463 & 0.07 & 3.8 & 4 \\
\hline 11348_7 & 153358 & 254.732264552 & -52.928718851 & 17.115 & 17.753 & 0.975 & 1.252 & 0.03 & 13.3 & - \\
\hline 11777_1 & 8568 & 254.921940390 & -52.633881686 & 17.234 & 17.765 & 0.989 & 1.090 & 0.02 & 7.1 & - \\
\hline 05369_3 & - & 254.487898227 & -52.688372708 & 17.095 & 17.769 & 1.086 & 1.366 & 0.0 & 10.4 & - \\
\hline 09457_3 & 53082 & 254.494573883 & -52.7023 & 17.060 & 17.794 & 1.148 & 1.443 & 0.0 & 10.0 & - \\
\hline 08455_7 & 150773 & 254.833791012 & -52.969794476 & 17.251 & 17.862 & 1.045 & 1.194 & 0.03 & 15.9 & - \\
\hline 03081_7 & 145976 & 254.826290498 & -53.048481819 & 17.175 & 17.900 & 1.016 & 1.354 & 0.05 & 20.5 & - \\
\hline 19255_4 & 85763 & 254.211076180 & -52.657335214 & 17.268 & 17.908 & 1.125 & 1.305 & 0.03 & 20.6 & - \\
\hline 09775_4 & 78525 & 254.349915968 & -52.647884819 & 17.143 & 17.922 & 1.065 & 1.461 & 0.06 & 15.7 & - \\
\hline 13658_1 & 9981 & 255.093499387 & -52.602477360 & 17.391 & 17.994 & 1.019 & 1.172 & 0.02 & 13.3 & - \\
\hline 01325_8 & 166592 & 254.899973622 & -52.990965132 & 17.233 & 17.999 & 1.146 & 1.451 & 0.03 & 17.6 & - \\
\hline 18991_5 & 109836 & 254.367964056 & -53.015663745 & 17.317 & 18.012 & 0.941 & 1.295 & 0.05 & 23.5 & - \\
\hline 10982_3 & 54387 & 254.462850556 & -52.575056451 & 17.415 & 18.018 & 0.926 & 1.165 & 0.01 & 13.8 & - \\
\hline 00690_2 & 23539 & 254.688379093 & -52.810472274 & 17.482 & 18.024 & 0.942 & 1.127 & 0.03 & 6.8 & - \\
\hline 20550_4 & 86799 & 254.392718310 & -52.799923440 & 17.370 & 18.071 & 1.089 & 1.393 & 0.05 & 14.8 & - \\
\hline 13897_3 & 56875 & 254.458096857 & -52.673541223 & 17.199 & 18.083 & 1.339 & 1.650 & 0.02 & 11.6 & - \\
\hline 07665_3 & 51566 & 254.484439046 & -52.643798202 & 17.280 & 18.091 & - & 1.561 & 0.10 & 11.1 & - \\
\hline 29733_4 & - & 254.190912380 & -52.703553212 & 17.482 & 18.107 & 1.011 & 1.255 & 0.03 & 21.1 & - \\
\hline 13846_2 & 33961 & 254.709665037 & -52.629843248 & 17.590 & 18.207 & 1.312 & 1.447 & 0.09 & 5.2 & $39 *$ \\
\hline
\end{tabular}


F. De Marchi et al.: Variable stars in NGC 6253

Table A.8. continued.

\begin{tabular}{|c|c|c|c|c|c|c|c|c|c|c|}
\hline Star & ID & $\begin{array}{l}\alpha_{2000} \\
{[\mathrm{deg}]}\end{array}$ & $\begin{array}{l}\delta_{2000} \\
{[\mathrm{deg}]}\end{array}$ & $\begin{array}{c}\bar{R} \\
{[\mathrm{mag}]}\end{array}$ & $\begin{array}{c}\bar{V} \\
{[\mathrm{mag}]}\end{array}$ & $\begin{array}{l}\bar{B}-\bar{V} \\
{[\mathrm{mag}]}\end{array}$ & $\begin{array}{c}\bar{V}-\bar{I} \\
{[\mathrm{mag}]}\end{array}$ & $\begin{array}{c}\text { Ampl. } \\
{[R \text { mag] }}\end{array}$ & $\begin{array}{l}\text { Distance } \\
\text { [arcmin] }\end{array}$ & $\begin{array}{c}\text { MP } \\
\%\end{array}$ \\
\hline 14975_3 & 57806 & 254.465207600 & -52.558813145 & 17.437 & 18.222 & 1.029 & 1.534 & 0.03 & 14.3 & - \\
\hline 13353_2 & 33571 & 254.703562371 & -52.636643531 & 17.551 & 18.246 & 1.342 & 1.534 & 0.06 & 5.0 & - \\
\hline 13124_3 & 56195 & 254.483299511 & -52.762663916 & 17.572 & 18.265 & 1.039 & 1.374 & 0.04 & 10.9 & - \\
\hline 13434_1 & 9812 & 255.062229061 & -52.606358224 & 17.774 & 18.318 & 0.993 & 1.136 & 0.03 & 12.2 & - \\
\hline 21802_4 & 87749 & 254.356986645 & -52.710333125 & 17.572 & 18.356 & 1.115 & 1.501 & 0.07 & 15.0 & - \\
\hline 12097_1 & 8801 & 255.009255709 & -52.628736854 & 17.377 & 18.370 & 1.490 & 1.933 & 0.02 & 9.9 & - \\
\hline 10299_3 & 53801 & 254.619740373 & -52.629216703 & 17.646 & 18.376 & 0.997 & 1.390 & 0.05 & 7.3 & - \\
\hline 14735_2 & 34670 & 254.747409696 & -52.617616901 & 17.577 & 18.396 & 1.286 & 1.708 & 0.08 & 5.5 & $55^{*}$ \\
\hline 27639_5 & 116432 & 254.187363541 & -52.872168980 & 17.296 & 18.397 & 1.469 & 2.067 & 0.06 & 23.3 & - \\
\hline 06772_1 & 4940 & 254.886895659 & -52.713142090 & 17.822 & 18.398 & 0.902 & 1.177 & 0.03 & 4.2 & - \\
\hline 19951_6 & 134785 & 254.525836051 & -52.908359235 & 17.630 & 18.430 & 1.258 & 1.526 & 0.05 & 14.9 & - \\
\hline 11608_1 & 8447 & 254.917947764 & -52.636605888 & 17.854 & 18.534 & 1.122 & 1.376 & 0.02 & 6.9 & - \\
\hline 09052_8 & 171605 & 254.917280122 & -52.908454920 & 17.444 & 18.535 & 1.563 & 2.296 & 0.04 & 13.1 & - \\
\hline 14979_2 & 34867 & 254.849030021 & -52.614345138 & 18.002 & 18.543 & 0.995 & 1.219 & 0.04 & 6.3 & - \\
\hline 12738_3 & 55863 & 254.512236460 & -52.802176775 & 17.902 & 18.662 & 1.159 & 1.550 & 0.05 & 10.9 & - \\
\hline 18918_2 & 37919 & 254.700600310 & -52.561027356 & 18.152 & 18.711 & 1.018 & 1.237 & 0.03 & 9.2 & - \\
\hline 24649_5 & 114100 & 254.189949967 & -52.917769863 & 17.943 & 18.732 & 1.164 & 1.476 & 0.11 & 24.5 & - \\
\hline 06593_3 & 50722 & 254.611170110 & -52.574208558 & 17.971 & 18.769 & 1.256 & 1.600 & 0.04 & 9.9 & - \\
\hline 26330_5 & 115420 & 254.376146705 & -53.059943442 & 18.168 & 18.835 & 1.084 & 1.315 & 0.06 & 25.5 & - \\
\hline 14117_3 & 57064 & 254.451450095 & -52.648219022 & 18.142 & 18.878 & 1.080 & 1.371 & 0.02 & 12.2 & - \\
\hline 05648_1 & 4137 & 255.028308571 & -52.731 & 18.202 & 18.892 & 1.098 & 1.424 & 14 & 9.5 & - \\
\hline 20127_8 & 179813 & 255.087 & -53.019120980 & 18.112 & 18.909 & 1.234 & 1.593 & 0.06 & 21.9 & - \\
\hline 16573_1 & 12106 & 254.996 & -52.554918919 & 18.373 & 18.959 & 1.066 & 1.191 & 0.02 & 12.3 & - \\
\hline 11636_2 & 32228 & 254.741734822 & -52.659219201 & 18.365 & 18.977 & 0.929 & 1.335 & 0.08 & 3.1 & $59 *$ \\
\hline 27451_6 & 140166 & 254.475678573 & -53.046675113 & 18.195 & 19.017 & - & 1.526 & 0.11 & 22.9 & - \\
\hline $06375 \_3$ & 50562 & 254.441211579 & -52.593573009 & 18.230 & 19.031 & - & 1.498 & 0.02 & 13.8 & - \\
\hline 01310_1 & 969 & 255.078222174 & -52.798865187 & 18.062 & 19.047 & 1.434 & 1.971 & 0.02 & 12.4 & - \\
\hline 28655_4 & 92338 & 254.377913474 & -52.733330472 & 18.303 & 19.059 & 1.190 & 1.521 & 005 & 14.4 & - \\
\hline 15984_8 & 176509 & 254.969343828 & -52.845694875 & 18.307 & 19.060 & 1.061 & 1.438 & 0.32 & 10.9 & - \\
\hline 12691_7 & 154548 & 254.734581721 & -52.909483223 & 18.166 & 19.090 & 1.324 & 1.788 & 0.14 & 12.1 & - \\
\hline 01647_1 & 1220 & 255.065821964 & -52.793412800 & 18.584 & 19.152 & 1.466 & 1.394 & 0.25 & 11.9 & - \\
\hline 01613_1 & 1194 & 254.911113437 & -52.794320612 & 18.556 & 19.156 & 1.023 & 1.184 & 0.03 & 7.2 & - \\
\hline 08210_3 & 52021 & 254.504165099 & -52.817828273 & 18.254 & 19.217 & 1.404 & 1.892 & 0.02 & 11.7 & - \\
\hline 05077_2 & 27059 & 254.688821000 & -52.748940203 & 18.568 & 19.331 & 1.288 & 1.661 & .11 & 3.8 & $8^{*}$ \\
\hline 09250_3 & 52908 & 254.62 & -52.72 & 18.533 & 19.357 & 1.222 & 1.604 & 0 & 5.5 & - \\
\hline 23979_5 & 113600 & 254.2 & -53.020 & 18.792 & 19.408 & 0.863 & 1.151 & 0.2 & 25.7 & - \\
\hline 14471_2 & 34464 & 254.85 & -52.621082471 & 18.616 & 19.439 & 1.283 & 1.765 & 0.02 & 6.0 & - \\
\hline 17201_2 & 36599 & 254.73 & -52.583879199 & 18.757 & 19.465 & 1.250 & 1.516 & 0.0 & 7.6 & - \\
\hline 24598_1 & 17470 & 254.929651594 & -52.587111414 & 18.874 & 19.478 & - & 1.216 & 0.24 & 9.3 & - \\
\hline 09627_3 & 53226 & 254.412694161 & -52.686333200 & 18.679 & 19.555 & 1.240 & 1.694 & 0.03 & 13.1 & - \\
\hline 26411_4 & 90948 & 254.253347727 & -52.744245829 & 18.622 & 19.575 & 0.891 & 1.683 & 0.23 & 18.9 & - \\
\hline 00589_2 & 23458 & 254.699544546 & -52.812227878 & 18.971 & 19.592 & 1.004 & 1.294 & 0.15 & 6.7 & - \\
\hline 28662_5 & 117213 & 254.358583280 & -52.886139263 & 18.946 & 19.595 & 0.946 & 1.241 & 0.06 & 18.4 & - \\
\hline 27455_4 & 91537 & 254.191463335 & -52.580710386 & 18.589 & 19.608 & 1.613 & 2.095 & 0.06 & 22.4 & - \\
\hline 26830_5 & 115800 & 254.387815806 & -52.922615996 & 18.965 & 19.703 & 1.021 & 1.352 & 0.45 & 18.9 & - \\
\hline 16144_2 & 35806 & 254.693955617 & -52.598659003 & 19.116 & 19.862 & 1.248 & 1.630 & 0.04 & 7.2 & - \\
\hline 20250_3 & 62457 & 254.444505271 & -52.584388594 & 19.144 & 19.912 & 1.090 & 1.517 & 0.04 & 14.0 & - \\
\hline 23070_7 & 163512 & 254.736493407 & -53.006712137 & 19.212 & 19.914 & - & - & 0.19 & 17.9 & - \\
\hline 02473_4 & 73146 & 254.374110070 & -52.614649897 & 19.107 & 19.939 & 0.932 & 1.532 & 0.12 & 15.5 & - \\
\hline 00771_1 & 573 & 254.938952802 & -52.807392294 & 18.825 & 19.948 & 1.571 & 2.654 & 0.05 & 8.5 & - \\
\hline 08578_8 & 171254 & 254.92 & -52.923498624 & 760 & 20.005 & 1.649 & 3.091 & 0. & 14.1 & - \\
\hline 12662_3 & 55797 & 254.426410046 & -52.811994226 & 19.352 & 20.069 & 1.284 & 1.574 & 0.05 & 14.0 & - \\
\hline 25559_3 & 67209 & 254.542912244 & -52.723409001 & 20.016 & 20.211 & 0.421 & 0.567 & 0.9 & 8.3 & - \\
\hline $04463 \_2$ & 26579 & 254.650065716 & -52.757554855 & 19.549 & 20.277 & 1.036 & 1.598 & 0.15 & 5.3 & - \\
\hline 12510_1 & 9109 & 255.027339311 & -52.621361522 & 19.739 & 20.286 & 0.943 & 1.083 & 0.17 & 10.7 & - \\
\hline 04386_1 & 3242 & 255.007984854 & -52.750361102 & 19.667 & 20.406 & 1.146 & 1.469 & 0.11 & 9.0 & - \\
\hline 14012_2 & 34092 & 254.716283261 & -52.627553480 & 19.948 & 20.470 & 1.041 & 1.273 & 0.17 & 5.2 & $0^{*}$ \\
\hline 13389_2 & 33601 & 254.787481909 & -52.636082942 & 19.975 & 20.750 & 1.090 & 1.639 & 0.07 & 4.4 & - \\
\hline 15110_1 & 11044 & 255.007528010 & -52.578768176 & 19.721 & 20.818 & 1.582 & 2.311 & 0.09 & 11.6 & - \\
\hline 25217_1 & 17912 & 254.974258061 & -52.570400191 & 21.028 & 22.140 & - & 2.414 & 1.87 & 11.1 & - \\
\hline 03001_7 & 145910 & 254.811174622 & -53.049901469 & 20.984 & 22.227 & - & 2.289 & 0.76 & 20.5 & - \\
\hline 29653_2 & - & 254.703773386 & -52.552573888 & 18.810 & - & - & - & 1.96 & 9.7 & - \\
\hline 14937_7 & - & 254.837063947 & -52.876393346 & 22.493 & - & - & - & 1.19 & 10.4 & - \\
\hline 27889_1 & 19734 & 254.974993381 & -52.619214002 & 22.261 & - & - & - & 2.49 & 9.2 & - \\
\hline 22073_1 & - & 254.894797264 & -52.656268542 & 22.888 & - & - & - & 1.83 & 5.5 & 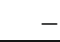 \\
\hline
\end{tabular}


Table A.9. List of binary systems (contact, detached and semi-detached binaries) located at less than $8^{\prime}$ from the centre which are considered as candidate members of NGC 6253.

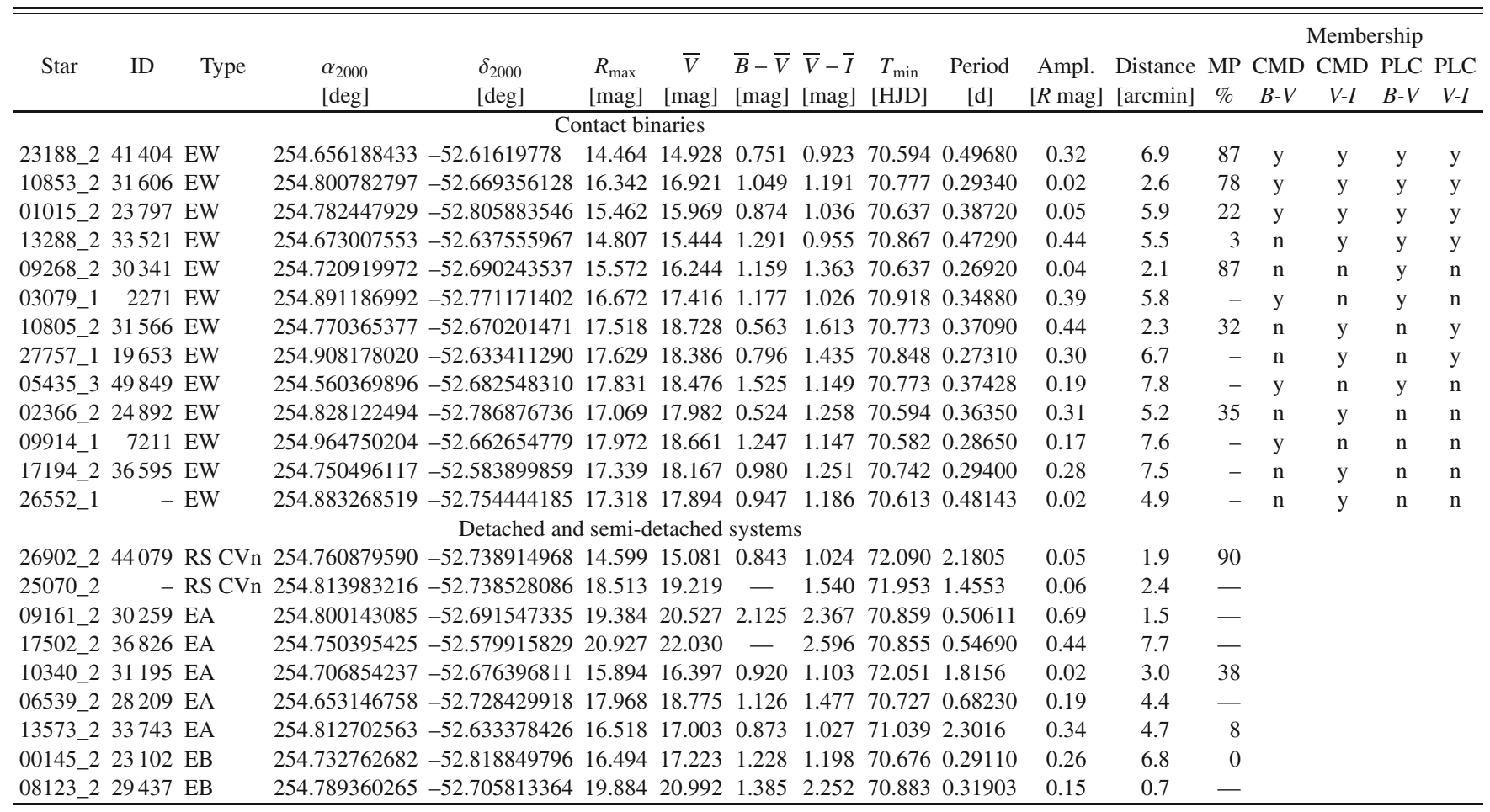

Table A.10. List of rotational and long-period variables located at less than $8^{\prime}$ from the centre which are considered to be candidate cluster members.

\begin{tabular}{|c|c|c|c|c|c|c|c|c|c|c|c|c|c|}
\hline Star & ID & Type & $\begin{array}{l}\alpha_{2000} \\
{[\mathrm{deg}]}\end{array}$ & $\begin{array}{l}\delta_{2000} \\
{[\mathrm{deg}]}\end{array}$ & $\begin{array}{c}\bar{R} \\
{[\mathrm{mag}]}\end{array}$ & $\begin{array}{c}\bar{V} \\
{[\mathrm{mag}]}\end{array}$ & $\begin{array}{l}\bar{B}-\bar{V} \\
{[\mathrm{mag}]}\end{array}$ & $\begin{array}{c}\bar{V}-\bar{I} \\
{[\mathrm{mag}]}\end{array}$ & $\begin{array}{c}T_{\max } \\
{[\text { HJD] }}\end{array}$ & $\begin{array}{l}\text { Period } \\
\text { [d] }\end{array}$ & $\begin{array}{l}\text { Ampl. } \\
{[R \mathrm{mag}]}\end{array}$ & $\begin{array}{l}\text { Distance } \\
\text { [arcmin] }\end{array}$ & $\begin{array}{c}\mathrm{MP} \\
\%\end{array}$ \\
\hline 09901_2 & 30842 & RO1 & 254.732275735 & 52.681556393 & 17.619 & 18.247 & 1.038 & 1.351 & 72.668 & 1.11842 & 0.08 & 2.1 & \\
\hline 11977_1 & 8713 & RO1 & 4.929865891 & 52.630746475 & 16.185 & 16.592 & 0.749 & 0.849 & 71.188 & 1.8424 & 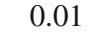 & & - \\
\hline 07392_1 & 5378 & 01 & 4.904680899 & 52.702647524 & 7.641 & 8.217 & 1.040 & 1.195 & 70.914 & 2.982 & & & 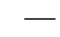 \\
\hline 14911_2 & 34814 & O1 & 4.721536966 & 52.615435459 & 17.702 & 18.299 & 1.053 & 1.212 & 70.902 & 1.5806 & 0. & & 63 \\
\hline 10372_2 & 1220 & RO1 & 54.679930555 & -52.675735040 & 17.650 & 18.317 & 1.080 & 1.456 & 72.957 & 2.735 & 0. & & 26 \\
\hline 15993_2 & 676 & RO1 & 4.846280438 & -52.600497324 & 18.851 & 19.741 & 1.462 & 1.844 & 71.766 & 3.151 & 0.0 & 7 & - \\
\hline 10042_2 & 30960 & RO1 & 4.772538141 & 52.679593806 & 5.946 & 16.379 & 0.872 & 1.029 & 71.445 & 3.13 & 0.0 & & 87 \\
\hline 11077_2 & 31786 & $\mathrm{O} 1$ & 278 & 52.666238861 & 6.725 & 17.281 & 0.978 & 1.194 & 70.801 & 5.1 & 0 . & & 71 \\
\hline 236_1 & 4548 & $\mathrm{O} 1$ & 476 & $52.721 \mathrm{C}$ & 6.148 & 16.614 & 0.865 & 0.972 & 72.207 & & & & - \\
\hline & 8498 & $\mathrm{O} 1$ & 851 & 4381 & 7.550 & 18.151 & 1.072 & 1.347 & & & & & 10 \\
\hline & 740 & $\mathrm{O} 1$ & 23 & 48 & 7.484 & & & 1.482 & & & & & 64 \\
\hline & 49473 & RO1 & & & & & & & & & & & - \\
\hline & & RO1 & & & & & & & & & & & - \\
\hline 8_2 & 40654 & 01 & & 56 & 57 & & & $2 .($ & & & & & - \\
\hline 7_2 & 28088 & 02 & & 27 & 70 & 76 & 0.9 & 1.130 & & & & & 85 \\
\hline & 1588 & $\mathrm{RO} 2$ & & 52. & 9.053 & 20.007 & 1.239 & 1.889 & 59 & 1.83 & & & - \\
\hline 15877_2 & - & Erupt. & 4.703 & -52.602 & 23.035 & - & - & - & - & - & & 6. & - \\
\hline 07619_2 & 29043 & LON & 961 & -52.712 & 17.009 & 17.559 & 0.960 & 1.147 & - & - & 0. & 1 & 7 \\
\hline 12230_2 & 32699 & LON & 044 & -52.65 & 17.029 & 17.748 & 1.192 & 1.463 & - & - & 0.0 & 3 & 4 \\
\hline 05077_2 & 059 & LON & 00 & -52.74 & 8.568 & 19.331 & 1.288 & 1.661 & - & - & 0.1 & & 8 \\
\hline 85_3 & 053 & LON & 1 & -52.71 & 6.004 & 16.539 & 0.892 & 1.031 & - & - & $0 .($ & & - \\
\hline 46_2 & 33961 & $\mathrm{ON}$ & 4.7 & -52.62 & 17.590 & 18.207 & 1.312 & 1.447 & - & - & 0.0 & 5. & 39 \\
\hline 14735_2 & 34670 & LON & 596 & -52.6 & 17.577 & 8.396 & 1.286 & 1.708 & - & - & 0.0 & & 55 \\
\hline 09250_3 & 52908 & LON & 52 & 52.72 & 18.533 & 19.357 & 1.222 & 1.604 & - & - & 0.1 & 5. & - \\
\hline 0_2 & 35074 & LON & & & 008 & 76 & 26 & 1.199 & - & - & & & 6 \\
\hline 12 & 23539 & LON & 993 & 274 & 17.482 & 18.024 & 0.942 & 1.127 & - & - & & 5.8 & 一 \\
\hline 11608_1 & 8447 & LON & 64 & & & & & & - & - & & & - \\
\hline & 16649 & LON & & & & & 0.856 & & - & - & & & - \\
\hline & 8568 & LON & & & & & 0.989 & & - & - & 0.0 & & - \\
\hline 13344_1 & 9747 & LON & & & 15.602 & & & 0.961 & - & - & 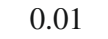 & & - \\
\hline & 551 & LON & & & & & & & - & - & & 7 & - \\
\hline & & L & & & 46 & & & & - & - & & 7. & - \\
\hline 17201_2 & 36599 & LON & 254.732771506 & -52.583879199 & 18.757 & 19.465 & 1.250 & 1.516 & - & - & 0.08 & 7.6 & - \\
\hline
\end{tabular}

